\title{
When Does a Dual Matrix Have a Dual Generalized Inverse?
}

\author{
Firdaus E. Udwadia
}

check for

updates

Citation: Udwadia, F.E. When Does a Dual Matrix Have a Dual Generalized Inverse? Symmetry 2021, 13, 1386. https://doi.org/10.3390/ sym13081386

Academic Editor: Paolo Emilio Ricci

Received: 24 June 2021

Accepted: 21 July 2021

Published: 30 July 2021

Publisher's Note: MDPI stays neutral with regard to jurisdictional claims in published maps and institutional affiliations.

Copyright: (C) 2021 by the author. Licensee MDPI, Basel, Switzerland. This article is an open access article distributed under the terms and conditions of the Creative Commons Attribution (CC BY) license (https:/ / creativecommons.org/licenses/by/ $4.0 /)$.
Aerospace and Mechanical Engineering, Civil and Environmental Engineering, Information and Operations Management, University of Southern California, Los Angeles, CA 90089, USA; fudwadia@usc.edu

\begin{abstract}
This paper deals with the existence of various types of dual generalized inverses of dual matrices. New and foundational results on the necessary and sufficient conditions for various types of dual generalized inverses to exist are obtained. It is shown that unlike real matrices, dual matrices may not have $\{1\}$-dual generalized inverses. A necessary and sufficient condition for a dual matrix to have a $\{1\}$-dual generalized inverse is obtained. It is shown that a dual matrix always has a $\{1\}-,\{1,3\}$-, $\{1,4\}-,\{1,2,3\}-,\{1,2,4\}$-dual generalized inverse if and only if it has a $\{1\}$-dual generalized inverse and that every dual matrix has a $\{2\}$ - and a $\{2,4\}$-dual generalized inverse. Explicit expressions, which have not been reported to date in the literature, for all these dual inverses are provided. It is shown that the Moore-Penrose dual generalized inverse of a dual matrix exists if and only if the dual matrix has a $\{1\}$-dual generalized inverse; an explicit expression for this dual inverse, when it exists, is obtained irrespective of the rank of its real part. Explicit expressions for the Moore-Penrose dual inverse of a dual matrix, in terms of $\{1\}$-dual generalized inverses of products, are also obtained. Several new results related to the determination of dual Moore-Penrose inverses using less restrictive dual inverses are also provided.
\end{abstract}

Keywords: dual matrices; dual generalized inverses; necessary and sufficient conditions for existence of dual generalized inverses; explicit expressions for various types of dual generalized inverses; explicit expressions for the dual generalized Moore-Penrose inverse using less restrictive dual generalized inverses

\section{Introduction}

The use of dual matrices has become common in various areas of science and engineering, such as the kinematic analysis and synthesis of machines and mechanisms, robotics and machine vision (see, for example, [1-3]). Over the last two decades, their use in science and engineering has increased significantly. In many forward and inverse problems in these areas, dual generalized inverses of dual matrices are sought and used. Because of familiarity with real matrices, many of the scientific and engineering communities that use dual matrices presuppose that generalized inverses of dual matrices always exist. It is only recently that the question of the existence of the Moore-Penrose dual generalized inverse has been raised and, in reference [4], it is shown that unlike the assured existence of a Moore-Penrose inverse of a real matrix, all dual matrices may not have Moore-Penrose (MP) dual inverses. This is demonstrated by the construction of an uncountably infinite set of dual matrices that are guaranteed to have no Moore-Penrose dual inverses. Reference [4] also shows that such dual matrices arise in practical inverse problems in kinematics and can lead to erroneous results when dealing with both experimental and simulated data. A recent finding shows that the reason why the Moore-Penrose dual generalized inverses of the dual matrices constructed in reference [4] do not exist is that these dual matrices do not have a $\{1\}$-dual generalized inverse [5].

Finding the generalized inverses of dual matrices is important for many problems that arise in various areas of science and engineering, where a dual matrix $\hat{A}$ often satisfies a given linear system of dual equations of the form $\hat{A} \hat{P}=\hat{Q}$ (see reference [5] for examples). Such linear systems are used in the so-called forward problems, in which the dual operator 
$\hat{A}$ and the dual matrix $\hat{P}$ are assumed to be sufficiently well known and the dual matrix $\hat{Q}$ is estimated, and in inverse problems, where the dual matrices $\hat{P}$ and $\hat{Q}$ are obtained from experiments and/or simulated data and the dual matrix operator $\hat{A}$ is required to be estimated. One can also envisage a variety of problems in which $\hat{A}$ and $\hat{Q}$ are given, but with one or both of them corrupted by measurement noise and a "good" approximate estimate of the solution $\hat{P}$ required. Various dual generalized matrices are useful for solving the analogs of the least-squares solutions of systems of real equations, minimum-norm solutions and minimum-norm least-squares solutions.

This paper investigates the existence of various types of dual generalized inverses that commonly arise in science and engineering. An $m$-by- $n$ dual matrix $\hat{A}=A+\varepsilon B$ is considered, where $\varepsilon$ is the hypercomplex unit basis, where $\varepsilon^{2}=0$ with $\varepsilon \neq 0$ for this unique nilpotent element, and the matrices $A$ and $B$ are real $m$-by- $n$ matrices. The matrices $A$ and $B$ are called the real (or primal) part and the dual part, respectively, of the matrix $\hat{A}$. We denote real matrices throughout by capital letters and hats on top of capital letters denote dual matrices. The four dual relations

$$
\begin{gathered}
\hat{A} \hat{G} \hat{A}=\hat{A} \\
\hat{G} \hat{A} \hat{G}=\hat{G} \\
\hat{A} \hat{G}=(\hat{A} \hat{G})^{T} \\
\hat{G} \hat{A}=(\hat{G} \hat{A})^{T}
\end{gathered}
$$

in which the dual matrix $\hat{G}=G+\varepsilon R$ is $n$-by- $m$, will be of central importance in this paper. These four successive relations will be referred to, respectively, as the first, second, third and fourth, Moore-Penrose (MP) dual conditions. When $B=0$, then $\hat{A}=A$ and $\hat{G}=G$ in Equations (1)-(4) and we obtain the well-known standard Moore-Penrose conditions for real matrices. Using the notation commonly followed for real matrices, we define a dual matrix $\hat{G}$ that satisfies the first MP dual condition (Equation (1)) to be the $\{1\}$-dual generalized inverse of the dual matrix $\hat{A}$ and denote it by $\hat{A}\{1\}$. If $\hat{G}$ satisfies the first three MP dual conditions (Equations (1)-(3)), it is called a $\{1,2,3\}$-dual generalized inverse of $\hat{A}$ and it is denoted by $\hat{A}^{\{1,2,3\}}$, and so on. We refer to the $\{1,2,3,4\}$-dual generalized inverse of $\hat{A}$ by $\hat{A}^{\{1,2,3,4\}}$; it is also called the Moore-Penrose dual generalized inverse (MPDGI) of $\hat{A}$.

While the apparent similarity between the conditions for dual matrices and real matrices is striking - one simply removes all the hats in Equations (1)-(4) to obtain their counterparts for real matrices-there are, however, significant differences. The most important of these is that while every real $m$-by- $n$ matrix $A$ has all kinds of generalized inverses, such as a $\{1\}-,\{1,2,3\}-,\{1,3,4\},\{1,2,4\}-,\{1,2,3,4\}$-generalized inverse, for an $m$-by- $n$ dual matrix none of these dual generalized inverses may exist.

The intent and scope of this paper is the development of new and foundational results on generalized inverses of dual matrices that would be of use in science and engineering with an eye to providing results that have analytical and especially computational value. The necessary and sufficient conditions for various types of dual generalized inverses to exist are obtained and, when these necessary and sufficient conditions are satisfied, explicit formulae for the dual generalized inverses are provided. While the interest from a science and engineering standpoint is often in $\{1,3\}$ - and $\{1,4\}$-dual inverses (the counterparts of the least-squares inverses for real matrices and minimum norm solutions of real matrix equations) and in the $\{1,2,3,4\}$-dual inverse (the counterpart of the Moore-Penrose inverse for real matrices), getting to the necessary and sufficient conditions for the existence of these dual inverses requires us to first obtain the corresponding conditions for other dual inverses to exist. These results are then systematically used to build-up the necessary and sufficient conditions for the existence of $\{1,3\}-,\{1,4\}$ - and $\{1,2,3,4\}$-dual inverses and other dual inverses as well. Several new properties of dual generalized inverses are obtained along the way, for example, formulae for the determination of the $\{1,2,3,4\}$-dual generalized inverse of a matrix, in terms of less restrictive inverses, such as $\{1,3\}$ - and 
$\{1,4\}$-dual generalized inverses. Throughout the paper the quintessential nature of $\{1\}$-dual generalized inverses in the context of the theory of dual generalized inverses developed here becomes apparent.

In Section 2 of the paper, some well-known preliminaries are given, which set the notation to be used throughout. The contributions of this paper to science and engineering are all presented in Section 3. Section 3.1 focuses on the existence of $\{1\}$ - and $\{2\}$-dual generalized inverses of $\hat{A}$ and obtains the necessary and sufficient conditions for a $\{1\}$-dual generalized inverse to exist; it is shown that all dual matrices have $\{2\}$-dual generalized inverses. In Section 3.2, the existence of $\{1,3\}$ - and $\{1,4\}$-dual generalized inverses is considered; Section 3.3 studies the existence of $\{2,4\}$-dual generalized inverses; Section 3.4 provides results for $\{1,2,3\}$ - and $\{1,2,4\}$-dual generalized inverses; Section 3.5 is concerned with the Moore-Penrose dual generalized inverse. Here, a new simple expression for the MPDGI of a dual matrix, irrespective of the rank of its real part, is obtained. In Section 3.6, somewhat remarkably, it is shown that explicit expressions for the Moore Penrose dual inverse of a dual matrix can be obtained in terms of only $\{1\}$-dual generalized inverses of its symmetric products. Several other new properties of dual generalized inverses and results, such as the formulae for the Moore-Penrose dual inverse in terms of $\{1,3\}$ - and $\{1,4\}$-dual generalized inverses, which are far less restrictive inverses, are obtained. The central importance of the $\{1\}$-dual generalized inverse in the development is highlighted. A more general existence proof than that given in reference [5] is presented, which gives as its byproduct the entire set of $\{1\}$-dual generalized inverses of a dual matrix. Throughout, for each type of dual generalized inverse investigated, the necessary and sufficient conditions for its existence are obtained along with explicit expressions for each type of dual generalized inverse. Section 4 provides the conclusions.

\section{Some Preliminaries}

This section establishes notation and provides several results that are well-known. They are placed together in this section because they are used in what follows.

We begin with finding the relations between the real matrices $A, B, G$, and $R$, so that for a given dual $m$-by- $n$ matrix $\hat{A}=A+\varepsilon B$, the dual matrix $\hat{G}=G+\varepsilon R$ satisfies the different Moore-Penrose (MP) dual conditions stated in Equations (1)-(4).

Lemma 1. The four MP dual conditions given in Equations (1)-(4) become the following.

(a) The first MP dual condition is equivalent to

$$
\begin{gathered}
A G A=A \\
B=B G A+A R A+A G B
\end{gathered}
$$

(b) The second MP dual condition is equivalent to

$$
\begin{gathered}
G A G=G, \\
R=R A G+G B G+G A R
\end{gathered}
$$

(c) The third MP dual condition is equivalent to

$$
\begin{gathered}
A G=(A G)^{T} \\
B G+A R=(B G+A R)^{T}
\end{gathered}
$$

(d) The fourth MP dual condition is equivalent to

$$
\begin{aligned}
G A & =(G A)^{T} \\
R A+G B & =(R A+G B)^{T}
\end{aligned}
$$


Proof. By substituting $\hat{A}=A+\varepsilon B$ and $\hat{G}=G+\varepsilon R$ in each of the MP dual conditions (1)-(4) and carrying out the computations, noting that $\varepsilon^{2}=0$, these results follow [4].

As mentioned before, a $\{1\}$-dual generalized inverse of $\hat{A}$ is defined as a dual matrix $\hat{G}$ that satisfies the first MP dual condition given in Equation (1) (or Equations (5) and (6)); a $\{2,3\}$-dual generalized inverse of $\hat{A}$ is defined as a dual matrix $\hat{G}$ that satisfies the second and third MP dual conditions given in Equations (1) and (3) (or Equations (7)-(10)), and so on.

Lemma 2. A dual generalized inverse of $\hat{A}=A+\varepsilon B$, if it exists, has the form $\hat{A}\{*\}=A^{\{*\}}+\varepsilon R$, where "** is any distinct sequence of the numbers $1,2,3,4$ and $A^{\{*\}}$ is the generalized inverse of the real part, $A$, of the dual matrix $\hat{A}$.

Proof. Seeing Equations (5), (7), (9) and (11) and removing the hats in Equations (1)-(4), so that we are dealing with real matrices now, the result is obvious.

Lemma 2 says that given a dual matrix $\hat{A}=A+\varepsilon B$, the real part $G$ of, say, its $\{1\}$-dual generalized inverse $\hat{A}^{\{1\}}$, if such an inverse exists, is simply a $\{1\}$-inverse of the real part, $A$, of $\hat{A}$. Similarly, $\hat{A}\{1,3\}$ must have the form $\hat{A}^{\{1,3\}}=A^{\{1,3\}}+\varepsilon R_{1}, A^{\{1,4\}}=A^{\{1,4\}}+\varepsilon R_{2}$, $\hat{A}^{\{1,2,3,4\}}=A^{\{1,2,3,4\}}+\varepsilon R_{3}$ and so on, if these dual generalized inverses exist. Thus, the real part of the $\left\{{ }^{*}\right\}$-dual generalized inverse of $\hat{A}$, if it exists, is simply $A^{\{*\}}$. Then, the main issue in finding a $\left\{{ }^{*}\right\}$-dual generalized inverse of a dual matrix $\hat{A}$, if it exists, is finding its dual part, $R$, since its real part is simply obtained by taking the real part, $A$, of $\hat{A}$ and finding its $\left\{{ }^{*}\right\}$-generalized inverse, which always exists and which we know how to obtain for any real matrix [6-9].

Lemma 3. To set our notation, we consider the singular value decomposition of a real $m$-by-n matrix $A$ of rank $r$ given by

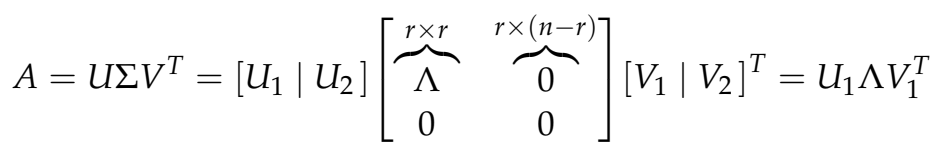

where $U$ and $V$ are, respectively, $m$-by- $m$ and $n$-by- $n$ orthogonal matrices and $\Lambda$ is an $r$-by- $r$ dimensional positive definite diagonal matrix. The matrices $U_{1}$ and $V_{1}$ are $m$-by- $r$ and $n$ by- $r$, respectively. Note that the submatrices $U_{1}$ and $V_{1}$ have columns that are orthonormal, so that the $r$-by-r matrices $U_{1}^{T} U_{1}=V_{1}^{T} V_{1}=I_{r} . U_{1}\left(V_{1}\right)$ is the orthogonal complement of $U_{2}\left(V_{2}\right)$.

Throughout this paper we will use the notation given in Equation (13) for the matrices $\Lambda, U, V$, and $U_{i}, V_{i}, i=1,2$, to refer to the singular value decomposition of the real part, $A$, of the dual matrix $\hat{A}=A+\varepsilon B$.

Using this notation, various generalized inverses of $A$ are obtained using the $n$-by- $m$ matrix:

$$
H=\left[V_{1} \mid V_{2}\right]\left[\begin{array}{cc}
\overbrace{\Lambda^{-1}}^{r \times r} & \overbrace{K}^{r \times(m-r)} \\
\underbrace{L}_{(n-r) \times r} & \underbrace{M}_{(n-r) \times(m-r)}
\end{array}\right]\left[U_{1} \mid \mathrm{U}_{2}\right]^{T}
$$

where the submatrices $K, L$ and $M$ are of the dimensions shown and the matrices $U_{i}, V_{i}, i=1,2$, come from the singular value decomposition of $A$ that is shown in Equation (13).

(1) To satisfy the first MP condition, the submatrices $K, L$ and $M$, can be any arbitrary matrices. Thus, every real matrix $A$ has at least one $\{1\}$-generalized inverse $A^{\{1\}}=H$, with $K, L$ and $M$ arbitrary. As seen, this dual generalized inverse is not unique. 
(2) To satisfy the second MP condition, we require that the submatrix $M=L \Lambda K$ and $K$ and $L$ can be any arbitrary matrices. Thus, $A^{\{2\}}=H$ with $K$ and $L$ arbitrary and $M=L \Lambda K$.

(3) To satisfy the third MP condition, we require that the submatrix $K=0$ and the matrices $L$ and $M$ can be arbitrary. Hence, $A^{\{3\}}=H$ with $L$ and $M$ arbitrary and $K=0$.

(4) To satisfy the fourth MP condition, we require that the submatrix $L=0$ and the matrices $K$ and $M$ can be arbitrary. Hence, $A^{\{4\}}=H$ with $K$ and $M$ arbitrary and $L=0$.

The four above-mentioned items can be used to obtain any desired $\left\{{ }^{*}\right\}$-generalized inverse of a real matrix $A$. For example, the $\{1,3\}$-generalized inverse of $A$ must be both a $\{1\}$ - and $\{3\}$-generalized inverse; therefore, it is given by:

$$
A^{\{1,3\}}=\left[V_{1} \mid V_{2}\right]\left[\begin{array}{cc}
\Lambda^{-1} & 0 \\
L & M
\end{array}\right]\left[U_{1} \mid \mathrm{U}_{2}\right]^{T}
$$

in which $L$ and $M$ are arbitrary matrices. Similarly,

$$
A^{\{1,4\}}=\left[V_{1} \mid V_{2}\right]\left[\begin{array}{ll}
\Lambda^{-1} & K \\
0 & M
\end{array}\right]\left[U_{1} \mid \mathrm{U}_{2}\right]^{T}
$$

in which $K$ and $M$ are arbitrary,

$$
A^{\{2,4\}}=\left[V_{1} \mid V_{2}\right]\left[\begin{array}{cc}
\Lambda^{-1} & K \\
0 & 0
\end{array}\right]\left[U_{1} \mid \mathrm{U}_{2}\right]^{T}
$$

in which $K$ is arbitrary, and

$$
A^{\{1,2,3,4\}}:=A^{+}=\left[V_{1} \mid V_{2}\right]\left[\begin{array}{ll}
\Lambda^{-1} & 0 \\
0 & 0
\end{array}\right]\left[U_{1} \mid \mathrm{U}_{2}\right]^{T}=V_{1} \Lambda^{-1} U_{1}^{T}
$$

and so on.

Every real matrix $A$ has, in general, a non-unique $A^{\{1\}}, A^{\{2\}}, A^{\{1,3\}}, A^{\{1,4\}}, A^{\{1,2,3\}}$, etc., and a unique $A^{\{1,2,3,4\}}$. We will denote $A^{\{1,2,3,4\}}$ by $A^{+}$, for short; it is also called the Moore-Penrose inverse of the real matrix $A$ and it is given by Equation (18). When $m=n$ and the matrix $A$ is nonsingular, then $A^{\{1,2,3,4\}}=A^{-1}$. It should be noted that the set of matrices $A^{\{2\}}$ (and $A^{\{2,4\}}$ ) provided in items (2) (and (4)) above give a subset of all the real matrices that satisfy only the second (and fourth) Moore-Penrose condition(s).

Proof. These results are well known (e.g., see [6,7]) and they are easy to prove.

As in Lemma 2, throughout this paper, we will refer to $A^{\{*\}}$ as the $\left.{ }^{*}\right\}$-inverse of the real part, $A$, of the dual matrix $\hat{A}=A+\varepsilon B$.

Lemma 4. Consider the matrix equation $A X B=C$, in which all the matrices are real. A solution, $X$, to this equation exists if and only if

$$
A A^{+} C B^{+} B=C
$$

and the solution $X$ to the matrix equation is explicitly given by

$$
X=A^{+} C B^{+}+Z-A^{+} A Z B B^{+}
$$

where the matrix $\mathrm{Z}$ is arbitrary.

Proof. See for example $[8,9]$ 


\section{Main Results}

In this section we obtain the necessary and sufficient conditions for various types of dual generalized inverses of an $m$-by- $n$ dual matrix $\hat{A}$ to exist and we also provide explicit expressions for them. These various dual generalized inverses are then used to obtain the necessary and sufficient conditions for the Moore-Penrose dual inverse to exist. It is shown that $\{2\}$ - and $\{2,4\}$-dual generalized inverses of a dual matrices always exist. Special attention is given to Moore-Penrose dual generalized inverses in Sections 3.5 and 3.6. In Section 3.6, the Moore-Penrose generalized inverse of a dual matrix $\hat{A}$ is obtained through the use of less restrictive dual generalized inverses. To the best of the author's knowledge, the results obtained here have not appeared before in the literature.

\subsection{On the Existence and Determination of a $\{1\}$ - and $\{2\}$-Dual Generalized Inverses of an $m-b y-n$ Dual Matrix $\hat{A}$}

From Lemma 2, we know that the $\{1\}$-dual inverse of the $m$-by-n matrix $\hat{A}=A+\varepsilon B$, if it exists, must have the form

$$
\hat{A}^{\{1\}}=A^{\{1\}}+\varepsilon R
$$

where $A^{\{1\}}$ is a $\{1\}$-generalized inverse of the real part, $A$, of the dual matrix $\hat{A}$. The matrix $A^{\{1\}}$ satisfies the condition given in Equation (5), i.e., $A A^{\{1\}} A=A$, and every real matrix $A$ has a $\{1\}$-generalized inverse, which is not unique, as seen in Lemma 3 . Our aim is to determine the necessary and sufficient conditions for an $n$-by- $m$ matrix $R$ to exist, so that it satisfies Equation (6).

The result proved here is more general than that given in reference [5] and it gives the entire set of matrices $R$ that yield the $\{1\}$-dual generalized inverse, which is an important generalization that is absent in reference [5]. Of similar importance, Equation (26) below lays down the notation that will be used in the several results that follow.

Result 1. The necessary and sufficient condition for an m-by-n dual matrix $\hat{A}=A+\varepsilon B$ to have a \{1\}-dual generalized inverse is that

$$
U_{2}^{T} B V_{2}=0
$$

where the matrices $U_{2}$ and $V_{2}$ are obtained from the singular value decomposition of the real part, $A$, of $\hat{A}$, using the notation established in Lemma 3 (see Equation (13)).

Proof. If $\hat{A}^{\{1\}}=G+\varepsilon R$ exists, then $\hat{A}^{\{1\}}$ satisfies the first MP dual condition given in Lemma 1, i.e., Equations (5) and (6). By Lemma 2, $G=A^{\{1\}}$, which always exists. Therefore, the necessary and sufficient condition for $\hat{A}^{\{1\}}$ to exist is that

$$
B=B A^{\{1\}} A+A R A+A A^{\{1\}} B
$$

Substituting the expressions for $A$ and $A^{\{1\}}$ given in Lemma 3, we obtain, from Equation (23), the relation

$$
B=B V\left[\begin{array}{cc}
I & 0 \\
L \Lambda & 0
\end{array}\right] V^{T}+U\left[\begin{array}{cc}
T & 0 \\
0 & 0
\end{array}\right] V^{T}+U\left[\begin{array}{cc}
I & \Lambda K \\
0 & 0
\end{array}\right] U^{T} B
$$

where $T:=\Lambda V_{1}^{T} R U_{1} \Lambda$. Note that $K, L$ and $M$ are arbitrary matrices (see Lemma 3 ) and the matrix $M$ does not show up in Equation (24).

Pre-multiplying both sides of Equation (24) by the orthogonal matrix $U^{T}$ and postmultiplying both sides by the orthogonal matrix $V$ give

$$
U^{T} B V=U^{T} B V\left[\begin{array}{cc}
I & 0 \\
L \Lambda & 0
\end{array}\right]+\left[\begin{array}{ll}
T & 0 \\
0 & 0
\end{array}\right]+\left[\begin{array}{cc}
I & \Lambda K \\
0 & 0
\end{array}\right] U^{T} B V
$$


Note that going from Equation (24) to Equation (25) is a reversible step. Since,

$$
U^{T} B V=\left[\begin{array}{l}
U_{1}^{T} B \\
U_{2}^{T} B
\end{array}\right]\left[\begin{array}{ll}
V_{1} V_{2}
\end{array}\right]=\left[\begin{array}{ll}
\overbrace{U_{1}^{T} B V_{1}}^{P} & \overbrace{U_{1}^{T} B V_{2}}^{Q} \\
\underbrace{U_{2}^{T} B V_{1}}_{S} & \underbrace{U_{2}^{T} B V_{2}}_{W}
\end{array}\right]:=\left[\begin{array}{cc}
\overbrace{P}^{r \times r} & \overbrace{Q}^{r \times(n-r)} \\
\underbrace{S}_{(m-r) \times r} & \underbrace{W}_{(m-r) \times(n-r)}
\end{array}\right]
$$

Equation (25) reduces to

$$
\left[\begin{array}{ll}
T & 0 \\
0 & 0
\end{array}\right]=\left[\begin{array}{cc}
-Y & -\Lambda K W \\
-W L \Lambda & W
\end{array}\right]
$$

where the $r$-by- $r$ matrix $Y=P+Q L \Lambda+\Lambda K S$. Equating the block sub-matrices on both sides gives

$$
\begin{gathered}
W=U_{2}^{T} B V_{2}=0 \\
V_{1}^{T} R U_{1}=-\Lambda^{-1} Y \Lambda^{-1}=-\Lambda^{-1}(P+Q L \Lambda+\Lambda K S) \Lambda^{-1}:=C_{1}
\end{gathered}
$$

Since each of the steps from Equation (23) to Equations (28) and (29) are reversible, we showed that the satisfaction of Equation (23) is equivalent to the two conditions given in Equations (28) and (29). Alternatively, the necessary and sufficient condition for $\hat{A}^{\{1\}}$ to exist stated in Equation (23) is the same as that given in Equation (27). Hence, the two conditions in Equations (28) and (29) are then the necessary and sufficient conditions that must be satisfied for the matrix $\hat{A}$ to have a $\{1\}$-dual generalized inverse.

The first of these conditions places a constraint on the matrix $B$, the dual part of $\hat{A}$; the second necessary and sufficient condition informs us that the matrix $R$ must satisfy the equation $V_{1}^{T} R U_{1}=C_{1}$, for all matrices $L$ and $K$ (see Lemma 3 ). The question now is whether such a matrix $R$ always exists.

We next show that such a matrix $R$ that satisfies Equation (29) always exists. To ensure this, we need to simply show that $V_{1}^{T} R U_{1}=C_{1}$ is consistent for any $r$-by-r matrix $C_{1}$. The necessary and sufficient condition for this equation to be consistent (see Lemma 4 , Equation (19)) is

$$
V_{1}^{T}\left(V_{1}^{T}\right)^{+} C_{1}\left(U_{1}\right)^{+}\left(U_{1}\right)=C_{1}
$$

where $\mathrm{X}^{+}$stands for the Moore-Penrose inverse (which always exists) of the real matrix $X$. However, Equation (30) is always true, because the submatrices $V_{1}$ and $U_{1}$ have columns that are orthonormal; therefore, $V_{1}^{+}=V_{1}^{T}$ and $U_{1}^{+}=U_{1}^{T}$. It then follows that $V_{1}^{T}\left(V_{1}^{T}\right)^{+}=V_{1}^{T}\left(V_{1}^{+}\right)^{T}=V_{1}^{T} V_{1}=I_{r}$ and $U_{1}^{+} U_{1}=U_{1}^{T} U_{1}=I_{r}$ so that the left-hand side always computes to $C_{1}$. Hence, consistency of Equation (29) is assured and the explicit solution for $R$ is then (see Lemma 4, Equation (20))

$$
R=V_{1} C_{1} U_{1}^{T}+Z-V_{1} V_{1}^{T} Z U_{1} U_{1}^{T}
$$

where $Z$ is an arbitrary $n$-by- $m$ matrix.

Thus, since a matrix $R$ that satisfies Equation (29) can always be found, the necessary and sufficient condition for $\hat{A}^{\{1\}}$ to exist is that given in Equation (28), $U_{2}^{T} B V_{2}=0$.

Result 2. The set of $\{1\}$-dual generalized inverses, $\hat{A}^{\{1\}}$, of the $m$-by-n dual matrix $\hat{A}=A+\varepsilon B$ exists if and only if the condition $U_{2}^{T} B V_{2}=0$ is satisfied and it is explicitly given by

$$
\hat{A}^{\{1\}}=A^{\{1\}}+\varepsilon\left(V_{1} C_{1} U_{1}^{T}+Z-V_{1} V_{1}^{T} Z U_{1} U_{1}^{T}\right)
$$

where $C_{1}=-\left(\Lambda^{-1} P \Lambda^{-1}+\Lambda^{-1} Q L+K S \Lambda^{-1}\right)$ and $Z$ is an arbitrary $n$-by- $m$ matrix. The matrices $U_{i}, V_{i}, i=1,2$ and $\Lambda$ are obtained from the singular value decomposition of $A$ (Equation (13)), given in Lemma 3. The matrices $P=U_{1}^{T} B V_{1}, Q=U_{1}^{T} B V_{2}$ and $S=U_{2}^{T} B V_{1}$ 
are shown in Equation (26). The set of matrices $A^{\{1\}}$ is explicitly given by the right-hand side of Equation (14) in which $K, L$ and $M$ are arbitrary matrices (see Lemma 3 ).

Proof. Using Equations (21) and (31) the result follows. We note, from Equation (32), that for a given dual matrix $\hat{A}$, neither the real nor the dual part of $\hat{A}^{\{1\}}$ is unique. Furthermore, for each $L$ and $K$ matrix chosen to obtain $A^{\{1\}}$ (see Lemma 3), there is an infinite set of matrices $R$. When $U_{2}^{T} B V_{2}=0$, Equation (32) gives the entire set of $\{1\}$-dual generalized inverse matrices of $\hat{A}$.

Remark 1. Setting $Z=0$ in Equation (32), a subset of \{1\}-dual generalized inverses of $\hat{A}$ is given by

$$
\hat{A}^{\{1\}}=A^{\{1\}}-\varepsilon V_{1}\left(\Lambda^{-1} P \Lambda^{-1}+\Lambda^{-1} Q L+K S \Lambda^{-1}\right) U_{1}^{T}
$$

Furthermore, since the matrices $K, L$ and $M$ on the right-hand side of Equation (14) are arbitrary, they can be chosen to be zero to yield a $\{1\}$-dual generalized inverse of $\hat{A}$ as

$$
\hat{A}^{\{1\}}=A^{+}-\varepsilon A^{+} B A^{+}
$$

This follows from Equation (33), because

$$
\hat{A}^{\{1\}}=A^{+}-\varepsilon V_{1}\left(\Lambda^{-1} P \Lambda^{-1}\right) U_{1}^{T}=A^{+}-\varepsilon \underbrace{V_{1} \Lambda^{-1} U_{1}^{T}}_{A^{+}} B \underbrace{V_{1} \Lambda^{-1} U_{1}^{T}}_{A^{+}}
$$

The bracketed terms follow from Equation (18) in Lemma 3. Thus, Equation (34) provides a simple, easy-to-compute expression that can be used to obtain a $\{1\}$-dual generalized inverse of a dual matrix $\hat{A}$.

Remark 2. When the real part, $A$, of the $m$-by-n dual matrix $\hat{A}=A+\varepsilon B$ has either full row rank or full column rank, the condition $U_{2}^{T} B V_{2}=0$ is always satisfied. This is because when the rank of $A$ is $m$, then $U_{2}=0$, and when the rank of $A$ is $n$, then $V_{2}=0$. Hence, when the real part of the dual matrix $\hat{A}$ has full row rank or full column rank, $\hat{A}^{\{1\}}$ always exists. The $m$-by-n matrices $A$ and $B$ of the dual matrix $\hat{A}$ can be independent of each other.

It is only when $A$ is rank deficient- $\operatorname{rank}(A)=r<m, n$-that the dual matrix $\hat{A}\{1\}$ might not exist. In that case, for $\hat{A}^{\{1\}}$ to exist, the matrices $A$ and $B$ cannot be independently chosen. This is because $U_{2}^{T} B V_{2}$ must equal the zero matrix.

A geometrical interpretation of the condition, $U_{2}^{T} B V_{2}=0$, for the $\{1\}$-dual inverse of a matrix $\hat{A}$ to exist is that the matrix $B$ must map the subspace orthogonal to $V_{1}$ (which is $V_{2}$ ) to the subspace $U_{1}$ (which is orthogonal to $U_{2}$ ).

An alternative geometrical meaning of this condition that is perhaps easier to state, since it concerns only the null and column space (range) of $A$, is as follows. The condition demands that $B$ must map the null space of $A, \mathcal{N}(A)$-which is the column space of $V_{2}$-so that it is perpendicular to the column space of $U_{2}$. However, $U_{2}^{\perp}=U_{1}$ and the column space of $U_{1}$ is the column space of $A$, namely, $\mathcal{R}(A)$. Hence, the necessary and sufficient condition $U_{2}^{T} B V_{2}=0$ demands that $B: \mathcal{N}(A) \rightarrow \mathcal{R}(A)$, that is, the matrix $B$, which is the dual part of $\hat{A}$, must map the null space of $A$ to the range of $A$. This interpretation has the advantage of simplicity and can often be checked more easily.

Remark 3. The necessary and sufficient condition $U_{2}^{T} B V_{2}=0$ for a $\{1\}$-dual generalized inverse to exist requires that the matrix B must always be expressible as (see Lemma 4, Equation (20))

$$
B=Z-\left(U_{2} U_{2}^{T}\right) Z\left(V_{2} V_{2}^{T}\right)
$$

for some $m$-by- $n$ matrix $Z$. As before, the matrices $U_{2}$ and $V_{2}$ come from the singular value decomposition of the real part, $A$, of the $m$-by- $n$ dual matrix $\hat{A}$ (see Lemma 3, Equation (13)). 
The homogeneous matrix equation $U_{2}^{T} B V_{2}=0$ is obviously consistent and its solution is explicitly given by Equation (36), since $\left(U_{2}^{T}\right)^{+}=U_{2}$ and $\left(V_{2}^{T}\right)^{+}=V_{2}$.

As stated in the previous remark, Equation (36) shows that, if either $U_{2}=0$ or $V_{2}=0$, then the dual part, $B$, of the matrix $\hat{A}$ can be any arbitrary matrix. However, if neither $U_{2}$ nor $V_{2}$ is zero, then $B$ cannot be an arbitrary matrix that is independent of the real part, $A$, of $\hat{A}$ in order for $\hat{A}^{\{1\}}$ to exist. In that case, the set of matrices $B$ are given explicitly in Equation (36), for any arbitrary matrix $Z$.

Remark 4. If the $\{1\}$-dual generalized inverse $\hat{A}^{\{1\}}=G+\varepsilon R$ of the $m$-by-n matrix $\hat{A}=A+\varepsilon B$ exists, then the $\{1\}$-dual generalized inverse $\left(\hat{A}^{T}\right)^{\{1\}}$ of the matrix $\hat{A}^{T}=A^{T}+\varepsilon B^{T}$ also exists. Since the \{1\}-inverse of Â must satisfy Equations (5) and (6), we have

$$
\begin{gathered}
A G A=A \Rightarrow A^{T} G^{T} A^{T}=A^{T} \\
B=B G A+A R A+A G B \Rightarrow B^{T}=B^{T} G^{T} A^{T}+A^{T} R^{T} A^{T}+A^{T} G^{T} B^{T}
\end{gathered}
$$

But the two implications above, that follow from Equations (5) and (6), are exactly the two relations required for $G^{T}+\varepsilon R^{T}$ to be a $\{1\}$-dual generalized inverse of $\hat{A}^{T}=A^{T}+\varepsilon B^{T}$. In short, if $\hat{A}^{\{1\}}=G+\varepsilon R$ is a $\{1\}$-dual generalized inverse of $\hat{A}=A+\varepsilon B$, then $\left(\hat{A}^{T}\right)^{\{1\}}=G^{T}+\varepsilon R^{T}$ is a $\{1\}$-dual generalized inverse of $\hat{A}^{T}=A^{T}+\varepsilon B^{T}$.

As an alternative proof, for finding the $\{1\}$-dual generalized inverse of $\hat{A}^{T}=A^{T}+\varepsilon B^{T}$, we need to find the singular value decomposition of its real part, $A^{T}$, which is given by $A^{T}=V_{1} \Lambda U_{1}^{T}$ (see Lemma 3). From Result $1, \hat{A}^{T}$ has a $\{1\}$-dual generalized inverse if and only if $V_{2}^{T} B^{T} U_{2}=0 \Rightarrow U_{2}^{T} B V_{2}=0$. Therefore, the necessary and sufficient condition for $\hat{A}^{T}$ to have a $\{1\}$-dual inverse is the same as that for $\hat{A}$ to have a $\{1\}$-dual generalized inverse! Hence, if the $\{1\}$-dual generalized inverse of $\hat{A}$ exists, then the $\{1\}$-dual generalized inverse of $\hat{A}^{T}$ also exists and vice-versa. The previous proof has the advantage of giving us that $\left(\hat{A}^{T}\right)^{\{1\}}=\left(\hat{A}^{\{1\}}\right)^{T}$, where $\hat{A}^{\{1\}}$ is given in Equation (32).

We next turn to finding the $\{2\}$-inverse of an $m$-by- $n$ matrix $\hat{A}$. We show that a $\{2\}$ generalized inverse of a dual matrix always exists and provide an explicit equation for it.

Result 3. Every dual matrix $\hat{A}=A+\varepsilon B$ always has a $\{2\}$-dual generalized inverse. In the notation of Lemma 3 (Equation (13), a set of $\{2\}$-dual inverses of $\hat{A}$ is explicitly given by

$$
A^{\{2\}}=A^{+}-\varepsilon\left(V_{1} \Lambda^{-1} P \Lambda^{-1} U_{1}^{T}-Z+V_{1} V_{1}^{T} Z U_{1} U_{1}^{T}+V_{2} V_{2}^{T} Z U_{2} U_{2}^{T}\right)
$$

in which $Z$ is an arbitrary $n$-by- $m$ matrix and $P=U_{1}^{T} B V_{1}$. The matrices $U_{i}, V_{i}, i=1,2$, are obtained from the singular value decomposition (see Lemma 3 ) of the real part, $A$, of $\hat{A}$ and $A^{+}$is the Moore-Penrose inverse of $A$.

Proof. We know from Lemma 2 that $\hat{A}^{\{2\}}=G+\varepsilon R=A^{\{2\}}+\varepsilon R$. The necessary and sufficient condition (see Lemma 1) for $\hat{A}^{\{2\}}$ to exist is that the matrix $R$ must satisfy the second MP dual condition (Equation (8)), so that

$$
R=R A A^{\{2\}}+A^{\{2\}} B A^{\{2\}}+A^{\{2\}} A R
$$

From Lemma 2, $A^{\{2\}}$ is obtained from the right-hand side of Equation (14) with the matrices $L$ and $K$ arbitrary and $M=L \Lambda K$. In addition, using the singular value decomposition of $A$ given in Equation (13), after some simplification, the MP dual condition becomes:

$$
R=R U\left[\begin{array}{cc}
I & \Lambda K \\
0 & 0
\end{array}\right] U^{T}+V\left[\begin{array}{ll}
\Lambda^{-1} & K \\
L & M
\end{array}\right]\left[\begin{array}{ll}
P & Q \\
S & W
\end{array}\right]\left[\begin{array}{ll}
\Lambda^{-1} & K \\
L & M
\end{array}\right] U^{T}+V\left[\begin{array}{ll}
I & 0 \\
L \Lambda & 0
\end{array}\right] V^{T} R
$$


where we denoted $U^{T} B V$ by the right-hand side of Equation (26).

Denoting

$$
V^{T} R U=\left[\begin{array}{cc}
V_{1}^{T} R U_{1} & V_{1}^{T} R U_{2} \\
V_{2}^{T} R U_{1} & V_{2}^{T} R U_{2}
\end{array}\right]:=\left[\begin{array}{cc}
P_{1} & Q_{1} \\
S_{1} & W_{1}
\end{array}\right]
$$

and pre-multiplying Equation (39) by $V^{T}$ and post-multiplying it by $U$, we obtain the relation

$$
\left[\begin{array}{cc}
P_{1} & Q_{1} \\
S_{1} & W_{1}
\end{array}\right]=\left[\begin{array}{ll}
P_{1} & P_{1} \Lambda K \\
S_{1} & S_{1} \Lambda K
\end{array}\right]+\left[\begin{array}{ll}
J_{1} & J_{2} \\
J_{3} & J_{4}
\end{array}\right]+\left[\begin{array}{lc}
P_{1} & Q_{1} \\
L \Lambda P_{1} & L \Lambda Q_{1}
\end{array}\right]
$$

where $(M=L \Lambda K)$

$$
\begin{array}{ll}
J_{1}:=\left(\Lambda^{-1} P+K S\right) \Lambda^{-1}+\left(\Lambda^{-1} Q+K W\right) L, & J_{2}=J_{1} \Lambda K:=\left(\Lambda^{-1} P+K S\right) K+\left(\Lambda^{-1} Q+K W\right) M \\
J_{3}=L \Lambda J_{1}:=(L P+M S) \Lambda^{-1}+(L Q+M W) L, & J_{4}:=(L P+M S) K+(L Q+M W) M .
\end{array}
$$

Equating the upper corner block submatrices and the lower corner block submatrices on both sides of Equation (41), we obtain the two independent equations $(M=L \Lambda K)$ :

$$
\begin{gathered}
P_{1}:=V_{1}^{T} R U_{1}=-J_{1} \\
W_{1}:=V_{2}^{T} R U_{2}=S_{1} \Lambda K+L \Lambda Q_{1}+J_{4}:=C_{2}
\end{gathered}
$$

that must be satisfied, so that a $\{2\}$-dual generalized inverse exists. Equating the offdiagonal block submatrices on both sides of Equation (41) does not lead to new equations. Notice that, in Equation (43), the matrices $S_{1}$ and $Q_{1}$ given in Equation (40) also depend on $R$.

As before, the steps from Equation (38) to Equations (42) and (43) are reversible; therefore, the necessary and sufficient conditions for a $\{2\}$-dual generalized inverse to exist are that Equations (42) and (43) be satisfied. However, as we shall now show, these equations can always be satisfied by a suitable matrix $R$.

To show that solution matrices, $R$, of these two equations always exist, we can set the arbitrary matrices $K$ and $L$ equal to zero, so that $M=0$. This makes $A^{\{2\}}=A^{+}$(see Equation (18), Lemma 3), $J_{1}=\Lambda^{-1} P \Lambda^{-1}, J_{4}=0$ and $C_{2}=0$. Then, Equations (42) and (43) become

$$
V_{1}^{T} R U_{1}=-\Lambda^{-1} P \Lambda^{-1}
$$

From Lemma 4, the second equation above has the solution $R=Z_{1}-V_{2} V_{2}^{T} Z_{1} U_{2} U_{2}^{T}$, which, when substituted in the first equation, gives $V_{1}^{T} Z_{1} U_{1}=-\Lambda^{-1} P \Lambda^{-1}$, since $V_{1}^{T} V_{2}=0$. Again, using Lemma 4 , the solution of this equation is given by $Z_{1}=-V_{1}\left(\Lambda^{-1} P \Lambda^{-1}\right) U_{1}^{T}+Z-V_{1} V_{1}^{T} Z U_{1} U_{1}^{T}$, where $Z$ is an arbitrary $n$-by- $m$ matrix. Substituting for $Z_{1}$ in the expression for $R$ that we just obtained and noting $V_{2}^{T} V_{1}=0$, the result follows.

\subsection{On the Existence of $a\{1,3\}$-and $\{1,4\}$-Dual Generalized Inverses of an $m$-by-n Dual Matrix $\hat{A}$}

A $\{1,3\}$-dual generalized inverse, $\hat{G}=G+\varepsilon R$, of an $m$-by- $n$ matrix $\hat{A}$, if it exists, is defined as a dual matrix that satisfies the first and third MP dual conditions $\hat{A} G \hat{A}=\hat{A}$ and $\hat{A} \hat{G}=(\hat{A} \hat{G})^{T}$. These two relations are equivalent to the relation

$$
\hat{A}^{T} \hat{A} \hat{G}=\hat{A}^{T}
$$

Clearly if the two relations are satisfied then $\hat{A}=\hat{A} \hat{G} \hat{A}=(\hat{A} \hat{G})^{T} \hat{A}=\hat{G}^{T} \hat{A}^{T} \hat{A}$. Taking the transpose of this relation yields Equation (45). Conversely, if Equation (45) is true then taking its transpose we obtain $\hat{A}=(\hat{A} \hat{G})^{T} \hat{A}$ and post multiplying both sides by $\hat{G}$ shows that $\hat{A} \hat{G}$ is symmetric and $\hat{A} \hat{G}=(\hat{A} \hat{G})^{T}$. Substituting this relation in $\hat{A}=(\hat{A} \hat{G})^{T} \hat{A}$ gives $\hat{A} \hat{G} \hat{A}=\hat{A}$. 
A $\{1,4\}$-dual generalized inverse, $\hat{G}=G+\varepsilon R$, of $\hat{A}$, if it exists, is defined as a dual matrix that satisfies the two MP dual conditions $\hat{A} \hat{G} \hat{A}=\hat{A}$ and $\hat{G} \hat{A}=(\hat{G} \hat{A})^{T}$. It can be shown in a similar manner that these two relations are equivalent to the relation

$$
\hat{A} \hat{A}^{T} \hat{G}^{T}=\hat{A}
$$

when dealing with real matrices one simply removes the hats everywhere in the above discussion to find that the first and third MP conditions $A G A=A$ and $A G=(A G)^{T}$ are equivalent to the relation $A^{T} A G=A^{T}$. Thus, a matrix $A^{\{1,3\}}$ is a $\{1,3\}$-generalized inverse of a real matrix $A$ if and only if $A^{T} A A^{\{1,3\}}=A^{T}$. Similarly, the first and fourth MP conditions $A G A=A$ and $G A=(G A)^{T}$ are equivalent to the relation $A A^{T} G^{T}=A$. Thus, a matrix $A^{\{1,4\}}$ is a $\{1,4\}$-generalized inverse of a real matrix $A$ if and only if $A A^{T}\left(A^{\{1,4\}}\right)^{T}=A$.

Result 4. The necessary and sufficient condition for an $m-b y-n$ dual matrix $\hat{A}=A+\varepsilon B$ to have a \{1,3\}-dual generalized inverse (see Section 1 for the definition) is that

$$
U_{2}^{T} B V_{2}=0
$$

where the matrices $U_{2}$ and $V_{2}$ are obtained from the singular value decomposition of the real part, $A$, of $\hat{A}$, using the notation established in Lemma 3 (see Equation (13)).

Proof. By Lemma 2, $\hat{A}\{1,3\}=\hat{G}=G+\varepsilon R=A^{\{1,3\}}+\varepsilon R$ must satisfy Equation (45) which, upon carrying out the multiplications $\left(\varepsilon^{2}=0\right)$, gives the necessary and sufficient condition for $\hat{A}^{\{1,3\}}$ to exist as

$$
A^{T} A G+\varepsilon\left(B^{T} A G+A^{T} B G+A^{T} A R\right)=A^{T}+\varepsilon B^{T}
$$

which becomes

$$
\begin{gathered}
A^{T} A G=A^{T} \\
B^{T}=B^{T} A G+A^{T} B G+A^{T} A R .
\end{gathered}
$$

The first equality simply states that $G=A^{\{1,3\}}$, which always exists, so that $\hat{A}^{\{1,3\}}=A^{\{1,3\}}+\varepsilon R$, something we already knew from Lemma 2 . Using the notation in Lemma 3 and substituting for $A$ and $G$ from Equations (13) and (15) in Equation (49), we obtain the necessary and sufficient condition for $\hat{A}^{\{1,3\}}$ to exist as

$$
B^{T}=B^{T} U\left[\begin{array}{ll}
I & 0 \\
0 & 0
\end{array}\right] U^{T}+V\left[\begin{array}{cc}
\Lambda & 0 \\
0 & 0
\end{array}\right] U^{T} B V\left[\begin{array}{ll}
\Lambda^{-1} & 0 \\
L & M
\end{array}\right] U^{T}+V_{1} \Lambda^{2} V_{1}^{T} R
$$

where $L$ and $M$ are arbitrary matrices (see Lemma 3). Pre-multiplying both sides by $V^{T}$ and post-multiplying both sides by $U$ and using the notation in Equation (26) for $U^{T} B V$ give

$$
\left[\begin{array}{cc}
P^{T} & S^{T} \\
Q^{T} & W^{T}
\end{array}\right]=\left[\begin{array}{ll}
P^{T} & 0 \\
Q^{T} & 0
\end{array}\right]+\left[\begin{array}{cc}
Y_{1} & \Lambda Q M \\
0 & 0
\end{array}\right]+\left[\begin{array}{cc}
\Lambda^{2} V_{1}^{T} R U_{1} & \Lambda^{2} V_{1}^{T} R U_{2} \\
0 & 0
\end{array}\right]
$$

where $Y_{1}=\Lambda P \Lambda^{-1}+\Lambda Q L$.

Then, equating the submatrices on both sides gives us the relations

$$
\begin{gathered}
W=U_{2}^{T} B V_{2}=0 \\
V_{1}^{T} R U_{1}=-\left(\Lambda^{-1} P \Lambda^{-1}+\Lambda^{-1} Q L\right) \\
V_{1}^{T} R U_{2}=\Lambda^{-2} S^{T}-\Lambda^{-1} Q M
\end{gathered}
$$

Since the steps from Equation (49) to Equations (52)-(54) are reversible, Equations (52)-(54) are the necessary and sufficient conditions for $\hat{A}^{\{1,3\}}$ to exist. 
Equation (52) places a restriction on $B$, the dual part of $A$; Equations (53) and (54) demand that a matrix $R$ must exist such that they are satisfied.

The last two equations can be combined to read

$$
V_{1}^{T} R U=\left[-\left(\Lambda^{-1} P \Lambda^{-1}+\Lambda^{-1} Q L\right) \mid \Lambda^{-2} S^{T}-\Lambda^{-1} Q M\right]
$$

so that

$$
V_{1}^{T} R=\left[-\left(\Lambda^{-1} P \Lambda^{-1}+\Lambda^{-1} Q L\right) \mid \Lambda^{-2} S^{T}-\Lambda^{-1} Q M\right] U^{T}:=C_{3} .
$$

We now prove that the dual part, $R$, always exists so that Equation (55) is satisfied. This requires us to show that Equation (55) is consistent for all matrices $C_{3}$. From Lemma 4 , the necessary and sufficient condition for consistency is that

$$
V_{1}^{T}\left(V_{1}^{+}\right)^{T} C_{3}=C_{3}
$$

which is the case, since the columns of $V_{1}$ are orthonormal and $V_{1}^{+}=V_{1}^{T}$, so that $V_{1}^{T}\left(V_{1}^{+}\right)^{T}=I_{r}$. Hence, only the necessary and sufficient condition in Equation (52) remains.

Result 5. The set of $\{1,3\}$-dual generalized inverses, $\hat{A}^{\{1,3\}}$, of the $m$-by- $n$ dual matrix $\hat{A}=A+\varepsilon B$ exists if and only if the condition $U_{2}^{T} B V_{2}=0$ is satisfied and it is explicitly given by the set of matrices

$$
\hat{A}^{\{1,3\}}=A^{\{1,3\}}+\varepsilon\left[V_{1} C_{3}+\left(I-V_{1} V_{1}^{T}\right) Z\right]
$$

where $Z$ is an arbitrary $n$-by- $m$ matrix and $C_{3}=\left[-\left(\Lambda^{-1} P \Lambda^{-1}+\Lambda^{-1} Q L\right) \mid \Lambda^{-2} S^{T}-\right.$ $\left.\Lambda^{-1} Q M\right] U^{T}$. The matrices $U_{i}, V_{i}, i=1,2$ and $\Lambda$ are obtained from the singular value decomposition of $A$ with the notation given in Lemma 3. The matrix $P=U_{1}^{T} B V_{1}, Q=U_{1}^{T} B V_{2}$ and $S=U_{2}^{T} B V_{1} . A^{\{1,3\}}$ are explicitly given in Equation (15), in which $L$ and $M$ are arbitrary matrices.

Proof. On the right-hand side of Equation (57), $A^{\{1,3\}}$ is explicitly given in Equation (15), in which $L$ and $M$ are arbitrary matrices. The dual part of $\hat{A}^{\{1,3\}}$ is obtained by solving Equation (55), which gives (see Lemma 4)

$$
R=\left(V_{1}^{T}\right)^{+} C_{3}+\left[I-\left(V_{1}^{T}\right)^{+} V_{1}^{T}\right] Z
$$

where $\mathrm{Z}$ is an arbitrary $n$-by- $m$ matrix. Noting that $\left(V_{1}^{T}\right)^{+}=\left(V_{1}^{+}\right)^{T}=V_{1}$, the result follows.

Remark 5. Setting $Z=0$ in Equation (57) gives a subset of \{1,3\}-dual generalized inverses of $\hat{A}=A+\varepsilon B$, that is explicitly given by

$$
\hat{A}^{\{1,3\}}=A^{\{1,3\}}+\varepsilon V_{1}\left[-\left(\Lambda^{-1} P \Lambda^{-1}+\Lambda^{-1} Q L\right) \mid \Lambda^{-2} S^{T}-\Lambda^{-1} Q M\right] U^{T}
$$

where the matrices $\Lambda, U$, and $U_{i}, V_{i}, i=1,2$, are obtained from the singular value decomposition of $A$ with the notation given in Lemma 3 (Equation (13)). The matrix $P=U_{1}^{T} B V_{1}$, $Q=U_{1}^{T} B V_{2}$ and $S=U_{2}^{T} B V_{1}$. The set of matrices $A^{\{1,3\}}$ is explicitly given by the right-hand side of Equation (15), in which $L$ and $M$ are arbitrary matrices.

Furthermore, setting the arbitrary matrices $L, M$ and $Z$ to zero, Equation (59) reduces and we obtain a simple expression for a $\{1,3\}$-dual generalized inverse of $\hat{A}$ as

$$
\hat{A}^{\{1,3\}}=A^{+}+\varepsilon V_{1}\left[-\Lambda^{-1} P \Lambda^{-1} \Lambda^{-2} S^{T}\right] U^{T}
$$

Remark 6. The entire discussion in Remarks 2 and 3 is also applicable to the $\{1,3\}$-generalized of a dual matrix $\hat{A}$.

We next consider the $\{1,4\}$-dual generalized inverse of the $m$-by- $n$ matrix $\hat{A}=A+\varepsilon B$. 
Result 6. The necessary and sufficient condition for an m-by-n dual matrix $\hat{A}=A+\varepsilon B$ to have a $\{1,4\}$-dual generalized inverse is that

$$
U_{2}^{T} B V_{2}=0
$$

Proof. By Lemma 2, $\hat{A}\{1,4\}=G+\varepsilon R=A^{\{1,4\}}+\varepsilon R$. The equation $\hat{A} \hat{A}^{T} \hat{G}^{T}=\hat{A}$ (Equation (46)) is satisfied with $\hat{G}=\hat{A}^{\{1,4\}}$. Carrying out the multiplications, this equation gives the necessary and sufficient conditions for the existence of a $\{1,4\}$-dual generalized inverse as

$$
A A^{T} G^{T}+\varepsilon\left(B A^{T} G^{T}+A B^{T} G^{T}+A A^{T} R^{T}\right)=A+\varepsilon B
$$

Hence, $A A^{T} G^{T}=A$, which is what is already known (Lemma 2), namely, $G=A^{\{1,4\}}$ (see Equation (16)), and we obtain the necessary and sufficient condition for the existence of a $\{1,4\}$-dual generalized inverse to be

$$
B A^{T} G^{T}+A B^{T} G^{T}+A A^{T} R^{T}=B
$$

Substituting for $A$ and $A^{\{1,4\}}$ from Equations (13) and (16) and using the notation for $U^{T} B V$ given in Equation (26), this equation reduces, after some algebra, to

$$
\left[\begin{array}{ll}
P & 0 \\
S & 0
\end{array}\right]+\left[\begin{array}{cc}
\Lambda P^{T} \Lambda^{-1}+\Lambda S^{T} K^{T} & \Lambda S^{T} M^{T} \\
0 & 0
\end{array}\right]+\left[\begin{array}{cc}
\Lambda^{2} U_{1}^{T} R^{T} V_{1} & \Lambda^{2} U_{1}^{T} R^{T} V_{2} \\
0 & 0
\end{array}\right]=\left[\begin{array}{cc}
P & Q \\
S & W
\end{array}\right]
$$

From which we obtain

$$
\begin{gathered}
W=U_{2}^{T} B V_{2}=0 \\
R U_{1}=V\left[\begin{array}{c}
-\left(\Lambda^{-1} P \Lambda^{-1}+K S \Lambda^{-1}\right) \\
Q^{T} \Lambda^{-2}-M S \Lambda^{-1}
\end{array}\right]:=C_{4}
\end{gathered}
$$

in which $K$ and $M$ are arbitrary matrices. Since all the steps from Equation (61) to Equations (64) and (65) are reversible, Equations (64) and (65) give the necessary and sufficient conditions for a $\{1,4\}$-dual generalized inverse of $\hat{A}$ to exist.

Equation (65) demands that a matrix $R$ that satisfies it exists. However, one can always find a matrix $R$ so that equation (65) is satisfied. From Lemma 4 , the necessary and sufficient condition for its consistency is that $C_{4}\left(U_{1}\right)^{+} U_{1}=C_{4}$, which is true, since $\left(U_{1}\right)^{+}=U_{1}^{T}$, and $U_{1}^{T} U_{1}=I_{r}$. This leaves only the necessary and sufficient condition given in Equation (64). Hence, the result.

Result 7. The set of $\{1,4\}$-dual generalized inverses, $\hat{A}\{1,4\}$, of the $m$-by- $n$ dual matrix $\hat{A}=A+\varepsilon B$ exists if and only if the condition $U_{2}^{T} B V_{2}=0$ is satisfied and it is explicitly given by (see Lemma 4 )

$$
\hat{A}^{\{1,4\}}=A^{\{1,4\}}+\varepsilon\left\{V\left[\begin{array}{c}
-\Lambda^{-1} P \Lambda^{-1}-K S \Lambda^{-1} \\
Q^{T} \Lambda^{-2}-M S \Lambda^{-1}
\end{array}\right] U_{1}^{T}+Z\left(I-U_{1} U_{1}^{T}\right)\right\}
$$

in which the matrices $\Lambda, U_{i}, V_{i}, i=1,2$, come from the singular value decomposition of $A$, as shown in Lemma 3, $Z, K$ and $M$ are arbitrary matrices (Lemma 3 ) and the set of matrices $A^{\{1,4\}}$ is explicitly given in Equation (16). The matrix $P=U_{1}^{T} B V_{1}, Q=U_{1}^{T} B V_{2}$ and $S=U_{2}^{T} B V_{1}$.

Proof. The solution of Equation (65) is directly obtained as shown in Equation (66) by using Lemma 4.

Remark 7. Setting $Z=0$ in Equation (66), we obtain a simpler subset for $R$ and

$$
\hat{A}^{\{1,4\}}=A^{\{1,4\}}+\varepsilon V\left[\begin{array}{c}
-\left(\Lambda^{-1} P \Lambda^{-1}+K S \Lambda\right) \\
Q^{T} \Lambda^{-2}-M S \Lambda^{-1}
\end{array}\right] U_{1}^{T}
$$


Further, choosing $K$ and $M$ to be zero, we find that an even simpler expression for a $\{1,4\}$-dual generalized inverse of $\hat{A}$ is given by

$$
\hat{A}^{\{1,4\}}=A^{+}+\varepsilon V\left[\begin{array}{c}
-\Lambda^{-1} P \Lambda^{-1} \\
Q^{T} \Lambda^{-2}
\end{array}\right] U_{1}^{T}
$$

Remark 8. The contents of Remarks 2 and 3 are applicable to the $\{1,4\}$-dual generalized inverse.

We note that the condition $U_{2}^{T} B V_{2}=0$ is a necessary and sufficient condition for a $\{1\}-$, a $\{1,3\}$-, $\{1,4\}$-dual generalized inverse to exist of a dual matrix. Therefore, we have the following important result.

Result 8. A matrix $\hat{A}$ has $\{1,3\}$-and $\{1,4\}$-dual generalized inverses if and only if it has a $\{1\}$-dual generalized inverse.

The existence of any one of the three matrices $\hat{A}^{\{1\}}, \hat{A}^{\{1,3\}}$, or $\hat{A}^{\{1,4\}}$ guarantees the existence of the other two.

Proof. The result follows from Results 1, 4 and 6.

\subsection{On the Existence of a \{2,4\}-Dual Generalized Inverses of an $m$-by-n Dual Matrix $\hat{A}$}

While the $\{1,3\}$ - and $\{1,4\}$-dual generalized inverses of a dual matrix are useful in the solution of dual matrix equations, the $\{2,4\}$-generalized inverse is not used as often. We obtain the necessary and sufficient conditions for the existence of this inverse, since we will need it in what follows.

A dual matrix is defined to be a $\{2,4\}$-dual generalized of the $m$-by- $n$ matrix $\hat{A}=A+\varepsilon B$ when it satisfies the second and fourth MP dual conditions (see Equations (2) and (4)), namely, $\hat{A}^{\{2,4\}} \hat{A} \hat{A}^{\{2,4\}}=\hat{A}^{\{2,4\}}$ and $\hat{A}^{\{2,4\}} \hat{A}=\left(\hat{A}^{\{2,4\}} \hat{A}\right)^{T}$. In a manner analogous to the $\{1,3\}$-dual generalized inverse, these two MP dual conditions are equivalent to the condition

$$
\hat{A}^{T}\left(\hat{A}^{\{2,4\}}\right)^{T} \hat{A}^{\{2,4\}}=\hat{A}^{\{2,4\}}
$$

If the second and fourth MP dual conditions are satisfied, then substituting $\hat{A}^{\{2,4\}} \hat{A}=\left(\hat{A}^{\{2,4\}} \hat{A}\right)^{T}$ in the second MP condition gives Equation (69). Conversely, we show that if Equation (69) is satisfied, the second and fourth MP conditions are satisfied. This is because from Equation (69), we obtain $\hat{A}^{T}\left(\hat{A}^{\{2,4\}}\right)^{T} \hat{A}^{\{2,4\}} \hat{A}=\hat{A}^{\{2,4\}} \hat{A}$, showing that $\hat{A}^{\{2,4\}} \hat{A}$ is symmetric; hence $\hat{A}^{\{2,4\}} \hat{A}=\left(\hat{A}^{\{2,4\}} \hat{A}\right)^{T}$. Thus, the fourth MP condition is satisfied. Substituting this relation in Equation (69) gives the second MP dual condition.

Result 9. The set of $\{2,4\}$-dual generalized inverse $\hat{A}^{\{2,4\}}$ of the $m$-by-n matrix $\hat{A}=A+\varepsilon B$ always exists and is explicitly given by the relation

$$
\hat{A}^{\{2,4\}}=A^{\{2,4\}}+\varepsilon R
$$

where

$$
R=V\left[\begin{array}{c}
-\left(\Lambda^{-1} P \Lambda^{-1}+K S \Lambda^{-1}\right) \\
\left(Q^{T} \Lambda^{-1}+W^{T} K^{T}\right) \Lambda^{-1}
\end{array}\right] U_{1}^{T}+V_{2}\left(Q^{T} \Lambda^{-1}+W^{T} K^{T}\right) K U_{2}^{T}-V_{2} V_{2}^{T} Z U_{2} U_{2}^{T}+Z\left(I-U_{1} U_{1}^{T}\right)
$$

in which $Z$ and $K$ are arbitrary matrices. The matrices $P=U_{1}^{T} B V_{1}, Q=U_{1}^{T} B V_{2}$ and $W=U_{2}^{T} B V_{2}$. 
Proof. Expressing $\hat{A}^{\{2,4\}}=G+\varepsilon R=A^{\{2,4\}}+\varepsilon R$ (see Lemma 2) and carrying out the multiplication on the left-hand side, Equation (69), that gives the necessary and sufficient condition for $\hat{A}^{\{2,4\}}$ to exist, is

$$
A^{T} G^{T} G+\varepsilon\left(B^{T} G^{T} G+A^{T} R^{T} G+A^{T} G^{T} R\right)=G+\varepsilon R
$$

This yields $A^{T} G^{T} G=G$, which is equivalent to $G=A^{\{2,4\}}$, something we already know; then, the necessary and sufficient condition for a $\{2,4\}$-dual generalized inverse to exist is

$$
B^{T} G^{T} G+A^{T} R^{T} G+A^{T} G^{T} R
$$

Substituting the expressions for $A$ and $A^{\{2,4\}}$ from Equations (13) and (17) (see Lemma 3), Equation (72) can be rewritten as

$$
B^{T} U\left[\begin{array}{cc}
\Lambda^{-2} & \Lambda^{-1} K \\
K^{T} \Lambda^{-1} & K^{T} K
\end{array}\right] U^{T}+V\left[\begin{array}{cc}
\Lambda P_{1}^{T} \Lambda^{-1} & \Lambda P_{1}^{T} K \\
0 & 0
\end{array}\right] U^{T}+V\left[\begin{array}{ll}
I & 0 \\
0 & 0
\end{array}\right] V^{T} R=R
$$

where we used the notation for $V^{T} R U$, as given in Equation (40). The matrix $K$ is arbitrary.

Pre-multiplying Equation (73) by $V^{T}$ and post-multiplying it by $U$, upon using the notation in Equation (26) for $U^{T} B V$, we obtain

$$
\left[\begin{array}{cc}
P^{T} \Lambda^{-2}+S^{T} K^{T} \Lambda^{-1} & P^{T} \Lambda^{-1} K+S^{T} K^{T} K \\
Q^{T} \Lambda^{-2}+W^{T} K^{T} \Lambda^{-1} & Q^{T} \Lambda^{-1} K+W^{T} K^{T} K
\end{array}\right]+\left[\begin{array}{cc}
\Lambda P_{1}^{T} \Lambda^{-1} & \Lambda P_{1}^{T} K \\
0 & 0
\end{array}\right]+\left[\begin{array}{cc}
P_{1} & Q_{1} \\
0 & 0
\end{array}\right]=\left[\begin{array}{cc}
P_{1} & Q_{1} \\
S_{1} & W_{1}
\end{array}\right]
$$

Equating the submatrices on both sides yields the independent conditions

$$
\begin{gathered}
P_{1}=V_{1}^{T} R U_{1}=-\left(\Lambda^{-1} P \Lambda^{-1}+K S \Lambda^{-1}\right), \\
S_{1}=V_{2}^{T} R U_{1}=\left(Q^{T} \Lambda^{-1}+W^{T} K^{T}\right) \Lambda^{-1} \\
W_{1}=V_{2}^{T} R U_{2}=\left(Q^{T} \Lambda^{-1}+W^{T} K^{T}\right) K .
\end{gathered}
$$

Since the steps from Equation (72) to Equations (75)-(77) can be reversed, the last three equations are the necessary and sufficient conditions for a $\{2,4\}$-dual generalized inverse to exist.

Combining the first two Equations (75) and (76), we obtain

$$
R U_{1}=V\left[\begin{array}{c}
-\left(\Lambda^{-1} P \Lambda^{-1}+K S \Lambda^{-1}\right) \\
\left(Q^{T} \Lambda^{-1}+W^{T} K^{T}\right) \Lambda^{-1}
\end{array}\right]
$$

By Lemma 4, this equation is consistent and its solution for $R$ is given by

$$
R=V\left[\begin{array}{c}
-\left(\Lambda^{-1} P \Lambda^{-1}+K S \Lambda^{-1}\right) \\
\left(Q^{T} \Lambda^{-1}+W^{T} K^{T}\right) \Lambda^{-1}
\end{array}\right] U_{1}^{T}+Z_{1}\left(I-U_{1} U_{1}^{T}\right)
$$

where $Z_{1}$ is an arbitrary $n$-by- $m$ matrix.

Substituting this expression for $R$ in Equation (77) gives

$$
V_{2}^{T} Z_{1} U_{2}=\left(Q^{T} \Lambda^{-1}+W^{T} K^{T}\right) K
$$

which is consistent (see Lemma 4) and whose solution is

$$
Z_{1}=V_{2}\left(Q^{T} \Lambda^{-1} K+W^{T} K^{T} K\right) U_{2}^{T}+Z-V_{2} V_{2}^{T} Z U_{2} U_{2}^{T}
$$

where $Z$ is an arbitrary $n$-by- $m$ matrix. Using this expression for $Z_{1}$ in Equation (78), we finally obtain $R$, which reduces to

$$
R=V\left[\begin{array}{c}
-\left(\Lambda^{-1} P \Lambda^{-1}+K S \Lambda^{-1}\right) \\
\left(Q^{T} \Lambda^{-1}+W^{T} K^{T}\right) \Lambda^{-1}
\end{array}\right] U_{1}^{T}+Z_{1}-Z U_{1} U_{1}^{T}
$$




\subsection{On the Existence of the $\{1,2,3\}$ and $\{1,2,4\}$ Dual Generalized Inverse of an $m$-by-n Matrix $\hat{A}$}

The $\{1,2,3\}$-dual generalized inverse of the $m$-by- $n$ matrix $\hat{A}=A+\varepsilon B$ is defined as a dual matrix that satisfies the conditions for being a $\{1,3\}$-dual generalized inverse (Equations (5), (6), (9) and (10)) and those for being a $\{2\}$-dual generalized inverse of $\hat{A}$ (Equations (7) and (8)). The necessary and sufficient conditions for a $\{1,3\}$-dual generalized inverse to exist are given in Equations (52)-(54) and the necessary and sufficient conditions for being a $\{2\}$-inverse are given in Equations (42)-(43). This motivates the following result.

Result 10. $\{1,2,3\}$-dual generalized inverses of the matrix $\hat{A}=A+\varepsilon B$ exists if and only if

$$
U_{2}^{T} B V_{2}=0
$$

where $U_{2}$ and $V_{2}$ come from the singular value decomposition of the real part, $A$, of $\hat{A}$, as shown in Equation (13), using the notation given in Lemma 3.

We show that this is true and provide an explicit result for the $\{1,2,3\}$-dual generalized inverse next.

Result 11. The set of $\{1,2,3\}$-dual generalized inverses of a dual matrix $\hat{A}=A+\varepsilon B$ exists if and only if the condition $U_{2}^{T} B V_{2}=0$ and it is explicitly given by

$$
\hat{A}^{\{1,2,3\}}=A^{\{1,2,3\}}+\varepsilon R
$$

In which

$$
R=-V_{1}\left[-\left(\Lambda^{-1} P \Lambda^{-1}+\Lambda^{-1} Q L\right) \mid \Lambda^{-2} S^{T}\right] U^{T}+V_{2}\left(L \Lambda^{-1} S^{T}\right) U_{2}^{T}-\left(V_{2} V_{2}^{T}\right) Z\left(U_{2} U_{2}^{T}\right)+\left(I-V_{1} V_{1}^{T}\right) Z
$$

where matrices $Z$ and $L$ are arbitrary. The notation in Lemma 3 regarding the singular value decomposition of $A$ is used. The set of matrices $A^{\{1,2,3\}}$ is given by the right-hand side of Equation (14), in which $K$ and $M$ are set to zero (see Lemma 3). The matrices $U$, $\Lambda, U_{i}, V_{i}, i=1,2$, come from the singular value decomposition of $A$ given in Equation (13) and $P=U_{1}^{T} B V_{1}, Q=U_{1}^{T} B V_{2}$ and $S=U_{2}^{T} B V_{1}$.

Proof. We know that $\hat{A}\{1,2,3\}=A^{\{1,2,3\}}+\varepsilon R$. Moreover, when finding the $\{1,3\}$-dual generalized inverse, we used $\hat{A}^{\{1,3\}}$ for the real part of $\hat{A}^{\{1,3\}}$. In order to ensure that the real part of $\hat{A}^{\{1,2,3\}}$ is $A^{\{1,2,3\}}$, we must ensure that $M=0$ (see Lemma 2, for the general form of $A^{\{1,2,3\}}$ ). Setting $M=0$ in the necessary and sufficient conditions for a $\{1,3\}$-dual generalized inverse to exist given Equations (52)-(54), we obtain

$$
W=U_{2}^{T} B V_{2}=0, V_{1}^{T} R U_{1}=-\left(\Lambda^{-1} P \Lambda^{-1}+\Lambda^{-1} Q L\right) \text { and } V_{1}^{T} R U_{2}=\Lambda^{-2} S^{T}
$$

In addition, $A^{\{1,2,3\}}$ is required to be a $\{2\}$-dual generalized inverse and, again, we want the real part of $A^{\{2\}}$ to be $A^{\{1,2,3\}}$. To ensure this, we need to set the matrices $K$ and $M$ to zero in the necessary and sufficient conditions for a $\{2\}$-dual inverse to exist given in Equations (42) and (43). This gives the necessary and sufficient conditions for a $\{2\}$-dual inverse to exist that has $K=M=0$ as

$$
\begin{gathered}
V_{1}^{T} R U_{1}=-\left(\Lambda^{-1} P \Lambda^{-1}+\Lambda^{-1} Q L\right) \\
V_{2}^{T} R U_{2}=L \Lambda Q_{1}=L \Lambda\left(V_{1}^{T} R U_{2}\right)=L \Lambda^{-1} S^{T}
\end{gathered}
$$

Combining Equations (83) and (85), we see that the necessary and sufficient conditions to be satisfied by a $\{12,3\}$-dual generalized inverse are

$$
W=U_{2}^{T} B V_{2}=0
$$




$$
V_{1}^{T} R U_{1}=-\left(\Lambda^{-1} P \Lambda^{-1}+\Lambda^{-1} Q L\right), V_{1}^{T} R U_{2}=\Lambda^{-2} S^{T} \text { and } V_{2}^{T} R U_{2}=L \Lambda^{-1} S^{T}
$$

Equation (87) demands that an $\mathrm{R}$ must exist that satisfies the three relations in it. We now show that one can always find a matrix $R$ that satisfies these relations. Since the real part of $\hat{A}^{\{1,2,3\}}$ is $A^{\{1,2,3\}}$, which is known to exist for any real matrix $A$, our concern is to find the matrix $R$.

The first two equations in (87) can be re-written as

$$
V_{1}^{T} R=\left[-\left(\Lambda^{-1} P \Lambda^{-1}+\Lambda^{-1} Q L\right) \mid \Lambda^{-2} S^{T}\right] U^{T}
$$

which is consistent by Lemma 4 and whose solution is

$$
R=V_{1}\left[-\left(\Lambda^{-1} P \Lambda^{-1}+\Lambda^{-1} Q L\right) \mid \Lambda^{-2} S^{T}\right] U^{T}+\left(I-V_{1} V_{1}^{T}\right) Z_{1}
$$

where $Z_{1}$ is an arbitrary $n$-by- $m$ matrix. Substituting this expression for $R$ in the last equation in Equation (87), we obtain the consistent (see Lemma 4) equation for $Z_{1}$ as

$$
V_{2}^{T} R U_{2}=V_{2}^{T} Z_{1} U_{2}=L \Lambda^{-1} S^{T}
$$

whose solution is

$$
Z_{1}=V_{2}\left(L \Lambda^{-1} S^{T}\right) U_{2}^{T}+Z-V_{2} V_{2}^{T} Z U_{2} U_{2}^{T}
$$

where $Z$ is an arbitrary $n$-by- $m$ matrix. Using this expression in Equation (89), we obtain

$$
R=V_{1}\left[-\left(\Lambda^{-1} P \Lambda^{-1}+\Lambda^{-1} Q L\right) \mid \Lambda^{-2} S^{T}\right] U^{T}+Z_{1}-V_{1} V_{1}^{T} Z
$$

Since there always exist matrices $R$ so that the necessary and sufficient conditions given in Equation (85) are always satisfied, the only necessary and sufficient condition left is the one in Equation (86); hence, the result.

The set of matrices $\hat{A}^{\{1,2,3\}}$ are explicitly obtained. Similar to this result, we now show that the set of $\{1,2,4\}$-dual generalized inverse of $\hat{A}$ always exists if and only if the $\{1\}$-dual inverse exists.

A $\{1,2,4\}$-dual generalized inverse is defined as a dual matrix that satisfies the first, second and fourth MP dual conditions (See Lemma 1).

Result 12. The dual matrix $\hat{A}=A+\varepsilon B$ has a $\{1,2,4\}$-dual generalized inverse if and only if it has a $\{1\}$-dual generalized inverse. The $\{1,2,4\}$ generalized inverse is given by

$$
\hat{A}^{\{1,2,4\}}=A^{\{1,2,4\}}+\varepsilon R
$$

where $A^{\{1,2,4\}}$ is given by the right-hand side of Equation (14) with the matrices $L$ and $M$ equal to zero and

$$
R=V\left[\begin{array}{c}
-\left(\Lambda^{-1} P \Lambda^{-1}+K S \Lambda^{-1}\right) \\
Q^{T} \Lambda^{-2}
\end{array}\right] U_{1}^{T}+V_{2} Q^{T} \Lambda^{-1} K U_{2}^{T}-V_{2} V_{2}^{T} Z U_{2} U_{2}^{T}+Z\left(I-U_{1} U_{1}^{T}\right)
$$

in which $Z$ and $K$ are arbitrary matrices and $P=U_{1}^{T} B V_{1}, Q=U_{1}^{T} B V_{2}$ and $W=U_{2}^{T} B V_{2}$. The matrices $U_{i}, V_{i}, i=1,2$, and $V$ come from the singular value decomposition of $A$ (see Lemma 3).

Proof. The $\hat{A}^{\{1,2,4\}}$ is both a $\{2,4\}$-dual generalized inverse and a $\{1\}$-dual generalized inverse. Its real part must be $A^{\{1,2,4\}}$. Hence, the matrices $L$ and $M$ must be both zero (see Lemma 3). Being a $\{2,4\}$-dual generalized inverse, $\hat{A}\{1,2,4\}$ must satisfy the necessary and sufficient conditions in Equations (75)-(77). In addition, it must satisfy the necessary and sufficient conditions for being a $\{1\}$-dual generalized inverse, namely, Equations (28) and (29), with $L=0$. Equation (28) says that $W=0$ and Equation (29), with 
$L=0$, is the same as Equation (75). Hence, the necessary and sufficient conditions for a $\{1,2,4\}$-dual generalized inverse to exist are

$$
\begin{gathered}
W=U_{2}^{T} B V_{2}=0 \\
V_{1}^{T} R U_{1}=-\left(\Lambda^{-1} P \Lambda^{-1}+K S \Lambda^{-1}\right), \\
V_{2}^{T} R U_{1}=Q^{T} \Lambda^{-2} \\
V_{2}^{T} R U_{2}=Q^{T} \Lambda^{-1} K .
\end{gathered}
$$

The last three conditions demand that a matrix $R$ must exist that satisfies Equations (92)-(94). However, the last three equations were already considered and a set of matrices $R$ that satisfies them was obtained in Equation (71). However, we need to set the matrices $L$ and $W$ to zero in it. Performing this, Equation (71) then gives

$$
R=V\left[\begin{array}{c}
-\left(\Lambda^{-1} P \Lambda^{-1}+K S \Lambda^{-1}\right) \\
Q^{T} \Lambda^{-2}
\end{array}\right] U_{1}^{T}+V_{2} Q^{T} \Lambda^{-1} K U_{2}^{T}-V_{2} V_{2}^{T} Z U_{2} U_{2}^{T}+Z\left(I-U_{1} U_{1}^{T}\right)
$$

in which $Z$ and $K$ are arbitrary matrices and $P=U_{1}^{T} B V_{1}, Q=U_{1}^{T} B V_{2}$ and $W=U_{2}^{T} B V_{2}$.

This leaves Equation (91), namely, $U_{2}^{T} B V_{2}=0$, as the remaining necessary and sufficient condition for the existence of the $\{1,2,4\}$-dual generalized inverse. Hence, the result.

\subsection{On the Existence of the Moore-Penrose Dual Generalized Inverse of an m-by-n Matrix $\hat{A}$}

In this section we obtain the necessary and sufficient condition for the Moore-Penrose dual inverse (MPDGI) of a matrix $\hat{A}$ to exist; when this condition is satisfied, the explicit closed for expression for the MPDGI is obtained. The proof of this result can also be found in reference [5] and is given here just for completeness.

Result 13. If the MPDGI of the dual matrix Â exists, it is unique.

Proof. See reference [5].

We next provide the closed-form expression for the MPDGI of a dual matrix irrespective of the rank of its real part and state the following new result.

Result 14. A unique Moore-Penrose dual generalized inverse of the m-by-n matrix $\hat{A}=A+\varepsilon B$ exists if and only if $U_{2}^{T} B V_{2}=0$ and it is given explicitly by

$$
\hat{A}^{\{1,2,3,4\}}=A^{+}+\varepsilon V\left[\begin{array}{cc}
-\Lambda^{-1} P \Lambda^{-1} & \Lambda^{-2} S^{T} \\
Q^{T} \Lambda^{-2} & 0
\end{array}\right] U^{T}
$$

where $A^{+}$is the unique Moore-Penrose generalized inverse (see Equation (18)) of the matrix $A$ and $P=U_{1}^{T} B V_{1}, Q=U_{1}^{T} B V_{2}$ and $S=U_{2}^{T} B V_{1}$. The notation in Lemma 3 is used and the matrices $\Lambda, U, V$, come from the singular value decomposition of the real part, $A$, of $\hat{A}$, as shown in Equation (13).

Proof. By Lemma 2, the MPDGI of $\hat{A}$ must have the form

$$
\hat{A}^{\{1,2,3,4\}}=A^{+}+\varepsilon R
$$

In which the matrix $R$ must be such that $\hat{A}^{\{1,2,3,4\}}$ is both a $\{1,3\}$-dual generalized inverse and a $\{2,4\}$-dual generalized inverse. However, we already know the necessary and sufficient conditions needed to be satisfied for a matrix to be a $\{1,3\}$-dual inverse, except that, in Section 3.2, we took the real part of the generalized inverse to be $A^{\{1,3\}}$. All we need to do is replace $A^{\{1,3\}}$ throughout the proof in Result 3 with $A^{+}$. However, this simply 
requires us to set $L$ and $M$ to zero, as seen in Lemma 2 . Hence, the conditions for a $\hat{A}$ to have a $\{1,3\}$-dual inverse are simply obtained from Equations (52)-(54) by setting $L$ and $M$ to zero in these equations. Thus, we obtain

$$
W:=U_{2}^{T} B V_{2}=0, V_{1}^{T} R U_{1}=-\Lambda^{-1} P \Lambda^{-1} \text { and } V_{1}^{T} R U_{2}=\Lambda^{-2} S^{T}
$$

in which $P=U_{1}^{T} B V_{1}$ and $S=U_{2}^{T} B V_{1}$.

Similarly, the necessary and sufficient conditions needed for $\hat{A}$ to have a $\{2,4\}$-dual generalized inverse are obtained directly from those in Section 3.3 using Equations (75)-(77) and setting $K=0$. We then obtain

$$
V_{1}^{T} R U_{1}=-\Lambda^{-1} P \Lambda^{-1}, V_{2}^{T} R U_{1}=Q^{T} \Lambda^{-2} \text { and } V_{2}^{T} R U_{2}=0
$$

in which $Q=U_{1}^{T} B V_{2}$. The real (primal) part of this MPDGI, if it exists, must be $A^{+}$, so $\hat{A}^{\{1,2,3,4\}}=A^{+}+\varepsilon R$. We must then show that the relations in Equations (98) and (99) can always be solved to obtain $R$.

Equations (98) and (99) lead to five necessary and sufficient conditions for the existence of an MPDGI, which can be re-written more compactly as

$$
V^{T} R U=\left[\begin{array}{cc}
-\Lambda^{-1} P \Lambda^{-1} & \Lambda^{-2} S^{T} \\
Q^{T} \Lambda^{-2} & 0
\end{array}\right] \text { and } U_{2}^{T} B V_{2}=0
$$

The first matrix equation gives $R$ as

$$
R=V\left[\begin{array}{cc}
-\Lambda^{-1} P \Lambda^{-1} & \Lambda^{-2} S^{T} \\
Q^{T} \Lambda^{-2} & 0
\end{array}\right] U^{T}
$$

The matrix $\Lambda^{-1} P \Lambda^{-1}$ is $r$-by- $r, Q^{T} \Lambda^{-2}$ is $(n-r)$-by- $r$, the matrix $\Lambda^{-2} S^{T}$ is $r$-by- $(m-r)$.

Thus, $\hat{A}^{\{1,2,3,4\}}=A^{+}+\varepsilon R$, with $R$ given by Equation (101). By Result 13, we know it is the unique MPDGI of $\hat{A}$, as is also obvious from the right-hand side of Equation (101). Since such a matrix $R$ always exists, the necessary and sufficient condition for the MPDGI to exist is $U_{2}^{T} B V_{2}=0$.

We note that the condition $U_{2}^{T} B V_{2}=0$ is a necessary and sufficient condition for a dual matrix to have a $\{1\}$-, a $\{1,3\}-,\{1,4\}$ - and a (unique) Moore-Penrose dual generalized inverse. Therefore, using the least restrictive inverse, we have the following important result.

Result 15. The Moore-Penrose dual inverse (MPDGI) of the dual matrix Â exists if and only if a \{1\}-dual generalized inverse of a matrix $\hat{A}$ exists.

Proof. The result follows from Results 1 and 14 .

Result 16. An m-by-n dual matrix that has either full row rank or full column rank always has a unique Moore-Penrose dual generalized inverse.

Proof. Since the dual matrix has either full row or full column rank, by Remark 2, it always has an $\{1\}$-dual generalized inverse and, from Results 13 and 15, the assertion follows.

While the above result is known "in isolation", the reason why the presence of the full rank of $A$ permits the existence of the MPDGI is, to the best of the author's knowledge, not known. The deeper connections discovered here, in Results 14 and 15, show that the reason for this is that a dual matrix $\hat{A}$ has to have a $\{1\}$-dual generalized inverse for its MPDGI to exist. Moreover, when it does have a $\{1\}$-dual generalized inverse, its MPDGI is given by the simple explicit expression given in Equation (101) (see Result 14), whatever the rank of $A$ might be. Thus, what really matters is not whether or not $A$ has full rank, (which is the accepted criterion scientists and engineers use today) but whether or not $\hat{A}$ has a 
$\{1\}$-dual generalized inverse. A dual matrix $\hat{A}$ can have a real part that is rank deficient and yet have an MPDGI, provided it has a $\{1\}$-dual generalized inverse. As shown in Result 16 , it just so happens that when $A$ has full row (column) rank, it always has a $\{1\}$-dual generalized inverse (see Remark 2) and, therefore, an MPDGI.

The results here have thus answered a deeper question-can a dual matrix whose real part is rank deficient have an MPDGI? The answer is in the affirmative and we gave the explicit expression for the MPDGI in Result 14.

Remark 8. When the real part of the $m$-by-m dual matrix $\hat{A}$ has rank $m$, then, by Remark $2, \hat{A}$ has a \{1\}-dual generalized inverse (see Remark 2). Equation (101) simplifies to $R=-V \Lambda^{-1} P \Lambda^{-1} U^{T}=$ $-V \Lambda^{-1} U^{T} B V \Lambda^{-1} U^{T}=-A^{+} B A^{+}$, giving

$$
\hat{A}^{\{1,2,3,4\}}=A^{+}-\varepsilon A^{+} B A^{+}=A^{-1}-\varepsilon A^{-1} B A^{-1}
$$

The last equality follows, because $A$ is nonsingular; therefore, $A^{+}=A^{-1}$. We consider this result a bit further in the next section. This result is well-known and we see how easily it falls out of the general expression for the MPDGI given in Equation (101), that is valid irrespective of the rank of $A$. As seen from the right-hand side of the first equality in (102), $\hat{A}^{\{1,2,3,4\}}$ is unique, something we already know from Result 13 .

In fact, when the real part, $A$, of the $m$-by-m (square) dual matrix $\hat{A}=A+\varepsilon B$ is nonsingular, then the matrix in the second equality in Equation (102) can be thought of as the unique "inverse" of the dual matrix $\hat{A}$. For, the dual matrix,

$$
\hat{A}^{-1}=A^{-1}-\varepsilon A^{-1} B A^{-1}
$$

which is also the MPDGI of $\hat{A}$ (see Equation (102)), is the dual counterpart of the real (ordinary) matrix inverse, as can be seen by computing the quantities

$$
\begin{gathered}
\hat{A} \hat{A}^{-1}=(A+\varepsilon B)\left(A^{-1}-\varepsilon A^{-1} B A^{-1}\right)=I \\
\hat{A}^{-1} \hat{A}=\left(A^{-1}-\varepsilon A^{-1} B A^{-1}\right)(A+\varepsilon B)=I+\varepsilon A^{-1} B\left(I-A^{-1} A\right)=I
\end{gathered}
$$

Thus, we find that

$$
\hat{A} \hat{A}^{-1}=\hat{A}^{-1} \hat{A}=I
$$

which is analogous to the relation satisfied by inverses of real (ordinary) matrices.

We can then call square $(m-$ by- $m)$ dual matrices whose real parts are nonsingular dual invertible matrices.

Observe that the dual inverse (MPDGI), $\hat{A}^{-1}$, of a dual invertible matrix $\hat{A}$ is obtained in Equation (103) directly in terms of its real and dual parts and no singular value decompositions are involved. In addition, when $B=0$, so that $\hat{A}$ becomes real, the equation simply becomes an identity.

As shown above, proving the known result given in Equation (103) is indeed trivial. It is included here because we shall see, in Result 18, that it has a very useful application when we extend our investigation beyond dual invertible matrices, that is, from square dual matrices whose real parts are nonsingular to non-square dual matrices whose real parts have full rank. However, first, we give a result on the dual inverse of a product of dual invertible matrices, which is analogous to that for real matrices.

Result 17. Consider the $m$-by-m dual invertible matrices $\hat{A}_{i}=A_{i}+\varepsilon B_{i}, i=1,2, \ldots, n$. Then

$$
\left(\hat{A}_{1} \hat{A}_{2} \ldots \hat{A}_{n-1} \hat{A}_{n}\right)^{-1}=\hat{A}_{n}^{-1} \hat{A}_{n-1}^{-1} \ldots A_{2}^{-1} A_{1}^{-1}
$$

or

$$
\left(\hat{A}_{1} \hat{A}_{2} \ldots \hat{A}_{n-1} \hat{A}_{n}\right)^{\{1,2,3,4\}}=\hat{A}_{n}^{\{1,2,3,4\}} \hat{A}_{n-1}^{\{1,2,3,4\}} \ldots A_{2}^{\{1,2,3,4\}} A_{1}^{\{1,2,3,4\}}
$$


This result, as with real matrices, also makes the computation and algebraic manipulation of MPDGIs of products of dual invertible matrices easier.

Proof. It suffices to prove that $\left(\hat{A}_{1} \hat{A}_{2}\right)^{-1}=\hat{A}_{2}^{-1} \hat{A}_{1}^{-1}$. Let $\hat{A}_{i}^{-1}=A_{i}^{-1}-\varepsilon A_{i}^{-1} B_{i} A_{i}^{-1}, i=1$, 2.. Then, $\hat{A}_{1} \hat{A}_{2}=A_{1} A_{2}+\varepsilon\left(B_{1} A_{2}+A_{1} B_{2}\right)$ and, since $A_{1} A_{2}$ is square and nonsingular,

$$
\begin{aligned}
\left(\hat{A}_{1} \hat{A}_{2}\right)^{-1} & =\left(A_{1} A_{2}\right)^{-1}-\varepsilon\left(A_{1} A_{2}\right)^{-1}\left(B_{1} A_{2}+A_{1} B_{2}\right)\left(A_{1} A_{2}\right)^{-1} \\
& =A_{2}^{-1} A_{1}^{-1}-\varepsilon\left(A_{2}^{-1} A_{1}^{-1} B_{1} A_{1}^{-1}+A_{2}^{-1} B_{2} A_{2}^{-1} A_{1}^{-1}\right)
\end{aligned}
$$

which is the same as $\hat{A}_{2}^{-1} \hat{A}_{1}^{-1}$, if one multiplies the two terms out.

The general result for the MPDGI of an $m$ by $n$ dual matrix, so far, has been found utilizing the framework of singular value decomposition. The next result shows that one can determine the $\{1,2,3,4\}$-dual generalized of a matrix $\hat{A}=A+\varepsilon B$, whose real part, $A$, has full row(column) rank directly in terms of its real and dual parts- $A$ and $B$-just like we observed in the case of dual invertible matrices.

Result 18. Consider the $m$-by-n dual matrix $\hat{A}=A+\varepsilon B$ :

(i) When $\operatorname{Rank}(A)=m$, i.e., $A$ has full row rank; then,

$$
\begin{gathered}
\hat{A}^{\{1,2,3,4\}}=A^{T} C+\varepsilon\left[B^{T} C-A^{T} C D C\right], \text { where } C=\left(A A^{T}\right)^{-1} \text { and } \\
D=B A^{T}+A B^{T}
\end{gathered}
$$

(ii) when $\operatorname{Rank}(A)=n$, i.e., $A$ has full column rank; then,

$$
\begin{gathered}
\hat{A}\{1,2,3,4\}=E A^{T}+\varepsilon\left[E B^{T}-E F E A^{T}\right], \text { where } E=\left(A^{T} A\right)^{-1} \text { and } \\
F=B^{T} A+A^{T} B .
\end{gathered}
$$

Proof. We prove the result for when $A$ has full row rank. The $m$-by- $m$ dual matrix

$$
\hat{A} \hat{A}^{T}=(A+\varepsilon B)\left(A^{T}+\varepsilon B^{T}\right)=A A^{T}+\varepsilon \underbrace{\left(B A^{T}+A B^{T}\right)}_{D}:=A A^{T}+\varepsilon D .
$$

Since $A$ has rank $m$, the $m$-by- $m$ matrix $A A^{T}$ also has rank $m$ and, therefore, is nonsingular. Thus, $\hat{A} \hat{A}^{T}$ is a dual invertible matrix. Using Equation (103), we then obtain

$$
\left(\hat{A} \hat{A}^{T}\right)^{\{1,2,3,4\}}=\left(\hat{A} \hat{A}^{T}\right)^{-1}=\left(A A^{T}\right)^{-1}-\varepsilon\left(A A^{T}\right)^{-1} D\left(A A^{T}\right)^{-1}
$$

We prove below, in part (iii) of Result 20, that, if the MPDGI of $\hat{A}$ exists-we know, by Result 16, that it does, because it has full row rank-then $\hat{A}^{\{1,2,3,4\}}=\hat{A}^{T}\left(\hat{A} \hat{A}^{T}\right)^{\{1,2,3,4\}}$. Hence, we obtain

$$
\hat{A}^{\{1,2,3,4\}}=\hat{A}^{T}\left(\hat{A} \hat{A}^{T}\right)^{+}=\hat{A}^{T}[\underbrace{\left(A A^{T}\right)^{-1}}_{C}-\varepsilon\left(A A^{T}\right)^{-1} D\left(A A^{T}\right)^{-1}]=\hat{A}^{T}[C-\varepsilon C D C]
$$

which, upon substituting for $\hat{A}$, is the same as Equation (109).

In a similar manner, when $A$ has full column rank, its MPDGI exists (Result 16). By considering the $n$-by- $n$ matrix $\hat{A}^{T} \hat{A}$, whose real part, $A^{T} A$, is nonsingular, Equation (110) follows, by considering $\hat{A}^{\{1,2,3,4\}}=\left(\hat{A}^{T} \hat{A}\right)^{\{1,2,3,4\}} \hat{A}^{T}$, which is proved in the following section, in part (iii) of Result 20. 


\subsection{Other Explicit Results for the Moore-Penrose Dual Generalized Inverse of a Matrix $\hat{A}$}

In the previous section, it is shown that, while the $\{1,2,3,4\}$-dual generalized inverse of a dual matrix, when it exists, is unique, the $\{1,3\}$ - and $\{1,4\}$-dual generalized inverses are not unique (see Section 3.2, Results 5 and 7). This is because $\{1,3\}$ - and $\{1,4\}$-dual generalized inverses are less restrictive than the $\{1,2,3,4\}$-dual generalized inverse, in that they are defined as dual matrices that need to satisfy only two of the four dual MP conditions, while an MP-dual generalized inverse (MPDGI) is defined as a matrix that must satisfy all four of them.

In this section, we begin with a result that permits the determination of the unique $\{1,2,3,4\}$-dual generalized inverse of a dual matrix through the use of any (every) $\{1,3\}$ - and any (every) $\{1,4\}$-dual generalized inverse of the dual matrix.

Result 19. If and only if either $\hat{A}^{\{1\}}$, or $\hat{A}^{\{1,3\}}$, or $\hat{A}^{\{1,4\}}$ exists, the MPDGI of $\hat{A}=A+\varepsilon B$ exists and it is explicitly given by

$$
\hat{A}^{\{1,2,3,4\}}=\hat{A}^{\{1,4\}} \hat{A} \hat{A}^{\{1,3\}}
$$

Proof. From Result 8, if and only if either one of the three matrices $\hat{A}^{\{1\}}, \hat{A}^{\{1,3\}}, \hat{A}^{\{1,4\}}$ exists, then the other two exist. Using the properties of $\hat{A}^{\{1,3\}}$ and $\hat{A}^{\{1,4\}}$ in Equations (1)-(4), we show that $\hat{A}$ given in Equation (112) satisfied the four dual Moore-Penrose conditions as follows.

MP dual Condition 1: $\hat{A}\left(\hat{A}^{\{1,4\}} \hat{A} \hat{A}^{\{1,3\}}\right) \hat{A}=\hat{A} \hat{A}^{\{1,4\}} \hat{A}=\hat{A}$.

MP dual Condition 2: $\hat{A}^{\{1,4\}} \hat{A} \hat{A}^{\{1,3\}} A \hat{A}^{\{1,4\}} \hat{A} \hat{A}^{\{1,3\}}=\hat{A}^{\{1,4\}} \hat{A} \hat{A}^{\{1,3\}} \hat{A} \hat{A}^{\{1,3\}}=\hat{A}^{\{1,4\}} \hat{A} \hat{A}^{\{1,3\}}$.

MP dual Condition 3: $\left(\hat{A} \hat{A}^{\{1,4\}} \hat{A} \hat{A}^{\{1,3\}}\right)^{T}=\left(\hat{A} \hat{A}^{\{1,3\}}\right)^{T}=\hat{A} \hat{A}^{\{1,3\}}=\hat{A} \hat{A}^{\{1,4\}} \hat{A} \hat{A}\{1,3\}$.

MP dual Condition 4: $\left(\hat{A}^{\{1,4\}} \hat{A} \hat{A}^{\{1,3\}} \hat{A}\right)^{T}=\left(\hat{A}^{\{1,4\}} \hat{A}\right)^{T}=\hat{A}^{\{1,4\}} \hat{A}=\hat{A}^{\{1,4\}} \hat{A} \hat{A}^{\{1,3\}} \hat{A}$

Result 19 says the following: As stated before, $\{1,3\}$ - and $\{1,4\}$-dual generalized inverses of the matrix $\hat{A}$ (when they exist) are, in general, not unique. Therefore, one has a set of $\{1,3\}$ and another set of $\{1,4\}$-dual generalized inverses of $\hat{A}$. Yet, no matter which particular $\{1,3\}$-dual generalized inverse and which particular $\{1,4\}$-dual generalized inverse one chooses from these sets, the product shown on the right-hand side of Equation (112) always yields the unique MPDGI of $\hat{A}$. This result is analogous to that for real (ordinary) generalized inverses.

We now introduce the following Lemma, which will be used in the results that follow.

Lemma 5. For any dual matrix $\hat{A}=A+\varepsilon B$, the matrices $\hat{A}^{T} \hat{A}, \hat{A} \hat{A}^{T}$ and $\hat{A}^{T} \hat{A} \hat{A}^{T}$ always have $\{1\}$-dual generalized inverses and, therefore, also $\{1,2,3,4\}$-dual generalized inverses.

Proof. The matrix $\hat{A}^{T} \hat{A}=A^{T} A+\varepsilon\left(B^{T} A+A^{T} B\right):=C+\varepsilon D$. Using the singular value decomposition of $A$ given in Equation (13) for the real part, $C$, of the dual matrix $\hat{A}^{T} \hat{A}$, we obtain $C=V\left[\begin{array}{ll}\Lambda^{2} & 0 \\ 0 & 0\end{array}\right] V^{T}$, so that $C^{\{1\}}=V\left[\begin{array}{cc}\Lambda^{-2} & K \\ L & M\end{array}\right] V^{T}=\left[V_{1} \mid V_{2}\right]\left[\begin{array}{cc}\Lambda^{-2} & K \\ L & M\end{array}\right]\left[V_{1} \mid V_{2}\right]^{T}$, where $K, L$ and $M$ are arbitrary matrices (see Lemma 3 ).

Using Result $1, \hat{A}^{T} \hat{A}$ has a $\{1\}$-dual generalized inverse if and only if $V_{2}^{T} D V_{2}=0$, where $D$ is the dual part of $\hat{A}^{T} \hat{A}$. We show that this is always true. Since $D=B^{T} A+A^{T} B$,

$$
V_{2}^{T} D V_{2}=V_{2}^{T}\left(B^{T} A+A^{T} B\right) V_{2}=V_{2}^{T} B^{T}\left(A V_{2}\right)+\left(A V_{2}\right)^{T} B V_{2}
$$

Again, using the singular value decomposition of $A$, we obtain

$$
A V_{2}=U\left[\begin{array}{cc}
\Lambda & 0 \\
0 & 0
\end{array}\right]\left[\begin{array}{l}
V_{1}^{T} \\
V_{2}^{T}
\end{array}\right] V_{2}=U\left[\begin{array}{cc}
\Lambda & 0 \\
0 & 0
\end{array}\right]\left[\begin{array}{l}
0 \\
I
\end{array}\right]=U\left[\begin{array}{l}
0 \\
0
\end{array}\right]=0
$$


Using Equation (114) we see, from Equation (113), that $V_{2}^{T} D V_{2}=0$; hence, $\hat{A}^{T} \hat{A}$ has an MPDGI.

In a similar manner, noting that $A^{T} U_{2}=0$, it can be shown that the matrices $\hat{A} \hat{A}^{T}$ and $\hat{A}^{T} \hat{A} \hat{A}^{T}$ always have $\{1\}$-dual generalized inverses. Result 15 shows that their MPDGIs always exist.

We now use this Lemma to prove the following result.

Result 20. If the matrix $\hat{A}$ has a $\{1\}$-dual generalized inverse, then

$$
\begin{gathered}
\left(\hat{A}^{T}\right)^{+}=\left(\hat{A}^{+}\right)^{T} \\
\left(\hat{A} \hat{A}^{T}\right)^{+}=\left(\hat{A}^{+}\right)^{T} \hat{A}^{+}=\left(\hat{A}^{T}\right)^{+} \hat{A}^{+} \\
\hat{A}^{+}=\left(\hat{A}^{T} \hat{A}\right)^{+} \hat{A}^{T}=\hat{A}^{T}\left(\hat{A} \hat{A}^{T}\right)^{+}
\end{gathered}
$$

where we denoted the superscript $\{1,2,3,4\}$ by " + " to reduce clutter.

Proof. By Lemma 5, the $\{1\}$-dual generalized inverse of $\hat{A}^{T} \hat{A}$ always exists for all dual matrices $\hat{A}$ and its MPDGIs also exist. By Remark 4, since the $\{1\}$-dual generalized inverse of $\hat{A}$ exists, the $\{1\}$-dual generalized inverse of $\hat{A}^{T}$ also exists; hence, the MPDGIs of both $\hat{A}$ and $\hat{A}^{T}$ exist. The proof given in reference [5] then follows.

Remark 9. Lemma 5 asserts that the two matrices $\hat{A} \hat{A}^{T}$ and $\hat{A}^{T} \hat{A}$ always have a $\{1\}$-dual generalized inverse; therefore, by Result 15, they each also have Moore-Penrose dual generalized inverses. Hence, it may be erroneously thought that the two product matrices $\left(\hat{A}^{T} \hat{A}\right)^{+} \hat{A}^{T}$ and $\hat{A}^{T}\left(\hat{A} \hat{A}^{T}\right)^{+}$ in Equation (117) always give the MPDGI of $\hat{A}$. While these two product matrices always do exist, they do not equal $\hat{A}^{\{1,2,3,4\}}$ in general, and Equation (117) is not true in general. The result in Equation (117) is true if and only if a \{1\}-dual generalized inverse of $\hat{A}$ also exists and, therefore, if and only if an MPDGI of $\hat{A}$ exists (see the proof in reference [5]).

Lemma 6. If and only if $\hat{A}^{\{1\}}$ exists, then

$$
\begin{gathered}
\hat{X} \hat{A} \hat{A}^{T}=\hat{Y} \hat{A} \hat{A}^{T} \Leftrightarrow \hat{X} \hat{A}=\hat{Y} \hat{A} \\
\hat{A} \hat{A}^{T} \hat{X}^{T}=\hat{A} \hat{A}^{T} \hat{Y}^{T} \Leftrightarrow \hat{A}^{T} \hat{X}^{T}=\hat{A}^{T} \hat{Y}^{T} \\
\hat{X} \hat{A}^{T} \hat{A}=\hat{Y} \hat{A}^{T} \hat{A} \Leftrightarrow \hat{X} \hat{A}^{T}=\hat{Y} \hat{A}^{T} \\
\hat{A}^{T} \hat{A} \hat{X}^{T}=\hat{A}^{T} \hat{A} \hat{Y}^{T} \Leftrightarrow \hat{A} \hat{X}^{T}=\hat{A} \hat{Y}^{T}
\end{gathered}
$$

Notice that in each of the Equations (118) and (121), we are, so to speak, "cancelling" $\hat{A}^{T}$ on both sides of the equation which is prior to the implication sign to arrive at the corresponding equation after the implication sign. Thus, one can so to speak "cancel" $\hat{A}^{T}$ on both sides of $\hat{X} \hat{A} \hat{A}^{T}=\hat{Y} \hat{A} \hat{A}^{T}$ to obtain $\hat{X} \hat{A}=\hat{Y} \hat{A}$ ! Similarly, in each of the Equations (119) and (120), we are, so to speak, "cancelling" $\hat{A}$ on both sides in the first equation to arrive at the second that follows the implication sign. In Equation (120), we so to speak "cancel" $\hat{A}$ on both sides of $\hat{X} \hat{A}^{T} \hat{A}=\hat{Y} \hat{A}^{T} \hat{A}$ to arrive at the equivalence $\hat{X} \hat{A}^{T}=\hat{Y} \hat{A}^{T}$.

Proof. If $\hat{A}^{\{1\}}$ exists, then $\hat{A}^{\{1,3\}}$ and $\hat{A}^{\{1,4\}}$ must exist (see Section 3.2, Results 4 and 6).

Multiplying both sides of Equation (118) by $\left(\hat{A}^{\{1,4\}}\right)^{T}$, we obtain

$$
\begin{aligned}
\hat{X} \hat{A} \hat{A}^{T}\left(\hat{A}^{\{1,4\}}\right)^{T}=\hat{Y} \hat{A} \hat{A}^{T}\left(A^{\{1,4\}}\right)^{T} & \Rightarrow \hat{X} \hat{A}\left(\hat{A}^{\{1,4\}} \hat{A}\right)^{T}=\hat{Y} \hat{A}\left(\hat{A}^{\{1,4\}} \hat{A}\right)^{T} \\
& \Rightarrow \hat{X} \hat{A} \hat{A}^{\{1,4\}} \hat{A}=\hat{Y} \hat{A} \hat{A}^{\{1,4\}} \hat{A},
\end{aligned}
$$

from which the result follows. 
Similarly, multiplying both sides of Equation (120) by $\left(A^{\{1,3\}}\right)^{T}$ gives

$$
\begin{aligned}
\hat{X} \hat{A}^{T} \hat{A} \hat{A}^{\{1,3\}}=\hat{Y} \hat{A}^{T} \hat{A} \hat{A}^{\{1,3\}} & \Rightarrow \hat{X} \hat{A}^{T}\left(\hat{A} \hat{A}^{\{1,3\}}\right)^{T}=\hat{Y} \hat{A}^{T}\left(\hat{A} \hat{A}^{\{1,3\}}\right)^{T} \\
& \Rightarrow \hat{X} \hat{A}^{T}\left(\hat{A}^{\{1,3\}}\right)^{T} \hat{A}^{T}=\hat{Y} \hat{A}^{T}\left(\hat{A}^{\{1,3\}}\right)^{T} \hat{A}^{T}, \\
& \Rightarrow \hat{X} \hat{A}^{T}=\hat{Y} \hat{A}^{T}
\end{aligned}
$$

Implications in the reverse direction in Equations (118)-(121) are obvious.

Equations (119) and (121) are obtained by transposing Equations (118) and (120); they will be used later.

We use this lemma to prove the next result, in which we obtain formulae for the most restrictive dual generalized inverse - the $\{1,2,3,4\}$-dual generalized inverse-of a dual matrix $\hat{A}$ (which must satisfy all four dual MP conditions) by using only one of the least restrictive dual generalized inverses-\{1\}-dual generalized inverses. As seen in Result 2, in Section 3.1, \{1\}-dual generalized inverses of $\hat{A}$ are a set of (uncountably infinite) matrices.

Result 21. If and only if the matrix $\hat{A}$ has a $\{1\}$-dual generalized inverse, then

$$
\hat{A}^{\{1,2,3,4\}}=\hat{A}^{T}\left(\hat{A} \hat{A}^{T}\right)^{\{1\}} \hat{A}\left(\hat{A}^{T} \hat{A}\right)^{\{1\}} \hat{A}^{T}
$$

Proof. By Lemma 5, the two $\{1\}$-dual generalized inverses in Equation (122) always exist. Hence, $I \hat{A} \hat{A}^{T}=\hat{A} \hat{A}^{T}\left(\hat{A} \hat{A}^{T}\right)^{\{1\}} \hat{A} \hat{A}^{T}$ and, with $X=I$ and $\hat{Y}=\hat{A} \hat{A}^{T}\left(\hat{A} \hat{A}^{T}\right)^{\{1\}}$, in Lemma 6 (Equation (118)), which is only applicable when $A^{\{1\}}$ exists, we obtain (cancelling the $\hat{A}^{T}$ effectively on both sides)

$$
\hat{A}=\hat{A} \hat{A}^{T}\left(\hat{A} \hat{A}^{T}\right)^{\{1\}} \hat{A}
$$

Using $\hat{A}^{T} \hat{A} I=\hat{A}^{T} \hat{A}\left(\hat{A}^{T} \hat{A}\right)^{\{1\}} \hat{A}^{T} \hat{A}$, with $\hat{X}^{T}=I$ and $\hat{Y}^{T}=\left(\hat{A}^{T} \hat{A}\right)^{\{1\}} \hat{A}^{T} \hat{A}$, in Equation (121), we obtain

$$
\hat{A}=\hat{A}\left(\hat{A}^{T} \hat{A}^{T}\right)^{\{1\}} \hat{A}^{T} \hat{A}
$$

In addition, from $\hat{A} \hat{A}^{T} I=\hat{A} \hat{A}^{T}\left(\hat{A} \hat{A}^{T}\right)^{\{1\}} \hat{A} \hat{A}^{T}$, with $I=\hat{X}^{T}$ and $\hat{Y}^{T}=\left(\hat{A} \hat{A}^{T}\right)^{\{1\}} \hat{A}$, by using (119), we obtain

$$
\hat{A}^{T}=\hat{A}^{T}\left(\hat{A} \hat{A}^{T}\right)^{\{1\}} \hat{A} \hat{A}^{T} \Rightarrow \hat{A}=\hat{A} \hat{A}^{T}\left[\left(\hat{A} \hat{A}^{T}\right)^{\{1\}}\right]^{T} \hat{A}
$$

Lastly, from $I \hat{A}^{T} \hat{A}=\hat{A}^{T} \hat{A}\left(\hat{A}^{T} \hat{A}\right)^{\{1\}} \hat{A}^{T} \hat{A}$, with $\hat{X}=I$ and $\hat{Y}=\hat{A}^{T} \hat{A}\left(\hat{A}^{T} \hat{A}\right)^{\{1\}}$, by using Equation (120), we obtain

$$
\hat{A}^{T}=\hat{A}^{T} \hat{A}\left(\hat{A}^{T} \hat{A}\right)^{\{1\}} \hat{A}^{T} \Rightarrow \hat{A}=\hat{A}\left[\left(\hat{A}^{T} \hat{A}\right)^{\{1\}}\right]^{T} \hat{A}^{T} \hat{A}
$$

We now consider the four MP dual conditions that must be satisfied by the right-hand side of Equation (122) as follows.

MP dual Condition 1:

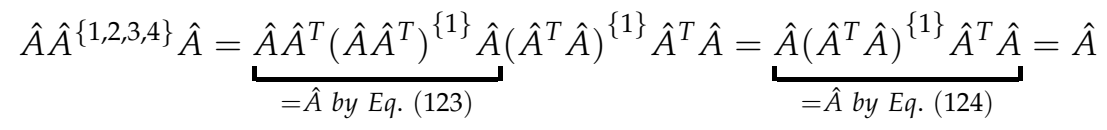

MP dual Condition 2:

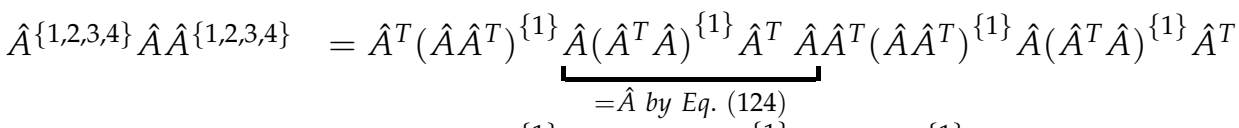

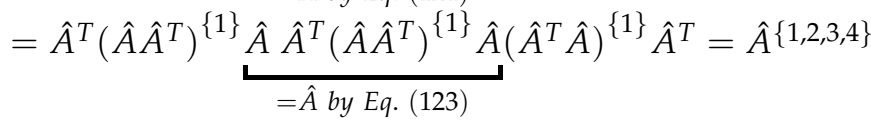


MP dual Condition 3:

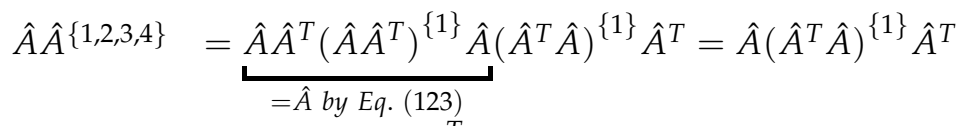

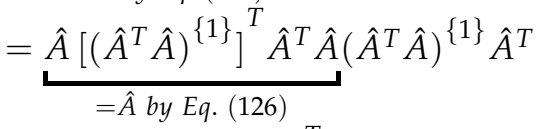

$$
\begin{aligned}
& =\left\{\hat{A}\left(\hat{A}^{T} \hat{A}\right)^{\{1\}} \hat{A}^{T}\right\}^{T}\left\{\hat{A}\left(\hat{A}^{T} \hat{A}\right)^{\{1\}} \hat{A}^{T}\right\},
\end{aligned}
$$

which is symmetric

MP dual Condition 4:

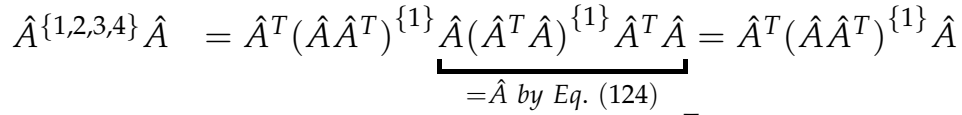

$$
\begin{aligned}
& =\hat{A}^{T}\left(\hat{A} \hat{A}^{T}\right)^{\{1\}} \underbrace{\hat{A} \hat{A}^{T}\left[\left(\hat{A} \hat{A}^{T}\right)^{\{1\}}\right]^{T} \hat{A}}_{=\hat{A} \text { by Eq. (125) }}=\left\{\hat{A}^{T}\left(\hat{A} \hat{A}^{T}\right)^{\{1\}} \hat{A}\right\}\left\{\hat{A}^{T}\left(\hat{A} \hat{A}^{T}\right)^{\{1\}} \hat{A}\right\}^{T},
\end{aligned}
$$

which is symmetric.

Remark 10. Recalling that the $\{1\}$-dual generalized inverse of a dual matrix is not unique, to obtain the unique MPDGI of $\hat{A}$, using Equation (122), any (every) \{1\}-dual generalized inverse $\hat{A} \hat{A}^{T}$ and any (every) $\{1\}$-dual generalized inverse of $\hat{A}^{T} \hat{A}$ can be used.

The next Lemma will be used to obtain the MPDGI of a dual matrix $\hat{A}$ using the $\{1\}$-dual generalized inverse of just a single matrix.

Lemma 7. If $\hat{A}$ has a $\{1\}$-dual generalized inverse, then the following relations are true.

$$
\begin{gathered}
\hat{A}=\hat{A} \hat{A}^{T}\left(\hat{A}^{T} \hat{A} \hat{A}^{T}\right)^{\{1\}} \hat{A}^{T} \hat{A} \\
\hat{A}^{T}=\hat{A}^{T} \hat{A} \hat{A}^{T}\left(\hat{A}^{T} \hat{A} \hat{A}^{T}\right)^{\{1\}} \hat{A}^{T}
\end{gathered}
$$

Proof. By Lemma 5, $\left(\hat{A}^{T} \hat{A} \hat{A}^{T}\right)^{\{1\}}$ always exists; therefore,

$$
\hat{A}^{T} \hat{A} \hat{A}^{T}=\hat{A}^{T} \hat{A} \hat{A}^{T}\left(\hat{A}^{T} \hat{A} \hat{A}^{T}\right)^{\{1\}} \hat{A}^{T} \hat{A} \hat{A}^{T}
$$

Furthermore, since $\hat{A}^{\{1\}}$ exists, Lemma 6 is applicable. Using the so-called "cancellation" observation in this lemma, we cancel the right-most $\hat{A}^{T}$ on either side of Equation (129) to obtain $\hat{A}^{T} \hat{A}=\hat{A}^{T} \hat{A} \hat{A}^{T}\left(\hat{A}^{T} \hat{A} \hat{A}^{T}\right)^{\{1\}} \hat{A}^{T} \hat{A}$. Again cancelling $\hat{A}^{T}$ and $\hat{A}$, respectively, on either side, we obtain the two relations $\hat{A}=\hat{A} \hat{A}^{T}\left(\hat{A}^{T} \hat{A} \hat{A}^{T}\right)^{\{1\}} \hat{A}^{T} \hat{A}$ and $\hat{A}^{T}=\hat{A}^{T} \hat{A} \hat{A}^{T}\left(\hat{A}^{T} \hat{A} \hat{A}^{T}\right)^{\{1\}} \hat{A}^{T}$, that are given in Equations (127) and (128).

Result 22. If $\hat{A}$ has a $\{1\}$-dual generalized inverse, then

$$
\hat{A}^{\{1,2,3,4\}}=\hat{A}^{T}\left(\hat{A}^{T} \hat{A} \hat{A}\right)^{\{1\}} \hat{A}^{T}
$$

Proof. We show that, by using Lemma 7, the four MP dual conditions are satisfied by the right-hand side of Equation (130) as follows.

MP dual Condition 1: By Equation (127),

$$
\hat{A} \hat{A}^{\{1,2,3,4\}} \hat{A}=\hat{A} \hat{A}^{T}\left(\hat{A}^{T} \hat{A} \hat{A}\right)^{\{1\}} \hat{A}^{T} \hat{A}=\hat{A} \text {. }
$$


MP dual Condition 2:

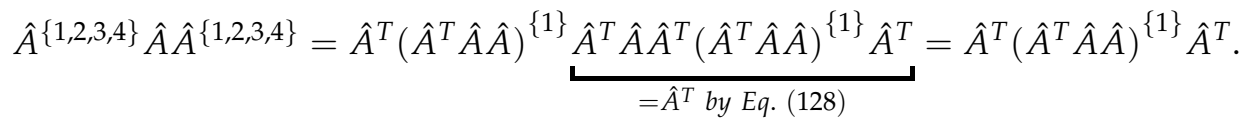

MP dual Condition 3:

$$
\begin{aligned}
\hat{A} \hat{A}^{\{1,2,3,4\}} & =\hat{A} \hat{A}^{T}\left(\hat{A}^{T} \hat{A} \hat{A}\right)^{\{1\}} \hat{A}^{T}=\hat{A} \hat{A}^{T}\left(\hat{A}^{T} \hat{A} \hat{A}\right)^{\{1\}} \underbrace{\hat{A}^{T} \hat{A}\left[\left(\hat{A}^{T} \hat{A} \hat{A}^{T}\right)^{\{1\}}\right]^{T} \hat{A} \hat{A}^{T}}_{=A^{T} \text { by Eq. (127) }} \\
& =\left\{\hat{A} \hat{A}^{T}\left(\hat{A}^{T} \hat{A} \hat{A}\right)^{\{1\}} \hat{A}^{T}\right\}\left\{\hat{A} \hat{A}^{T}\left(\hat{A}^{T} \hat{A} \hat{A}\right)^{\{1\}} \hat{A}^{T}\right\}^{T} \text {, which is symmetric. }
\end{aligned}
$$

MP dual Condition 4:

$$
\begin{aligned}
\hat{A}^{\{1,2,3,4\}} \hat{A} & =\hat{A}^{T}\left(\hat{A}^{T} \hat{A} \hat{A}\right)^{\{1\}} \hat{A}^{T} \hat{A}=\underbrace{\hat{A}^{T} \hat{A}\left[\left(\hat{A}^{T} \hat{A} \hat{A}^{T}\right)^{\{1\}}\right]^{T} \hat{A} \hat{A}^{T}\left(\hat{A}^{T} \hat{A} \hat{A}\right)^{\{1\}} \hat{A}^{T} \hat{A}}_{=A^{T} \text { from Eq. }(127)} \\
& =\left\{\hat{A}^{T}\left(\hat{A}^{T} \hat{A} \hat{A}\right)^{\{1\}} \hat{A}^{T} \hat{A}\right\}^{T}\left\{\hat{A}^{T}\left(\hat{A}^{T} \hat{A} \hat{A}\right)^{\{1\}} \hat{A}^{T} \hat{A}\right\}, \text { which is symmetric. }
\end{aligned}
$$

Lastly, we consider the $\{1,2\}$-dual generalized inverse of the matrix $\hat{A}$. It is shown that its existence is guaranteed by the existence of a $\{1\}$-dual generalized inverse of $\hat{A}$ and a simple way to obtain it from any (every) $\{1\}$-dual generalized inverse of $\hat{A}$ is presented.

Result 23. If Â has a \{1\}-dual generalized inverse, then

$$
\hat{A}^{\{1,2\}}=\hat{A}^{\{1\}} \hat{A} \hat{A}^{\{1\}}
$$

Proof. We show that the first two MP conditions are satisfied as follows.

MP dual Condition 1:

$$
\hat{A} \hat{A}^{\{1,2\}} \hat{A}=\underbrace{\hat{A} \hat{A}^{\{1\}}}_{\hat{A}} \hat{A} \hat{A}^{\{1\}} \hat{A}=\hat{A} \hat{A}^{\{1\}} \hat{A}=\hat{A} .
$$

MP dual Condition 2:

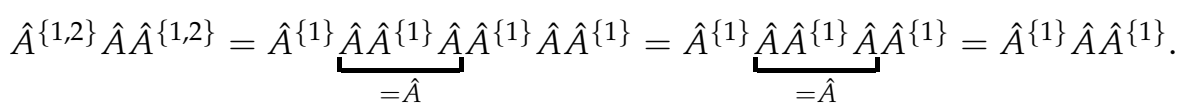

The expressions reported for the MPDGI of a dual matrix in Results 21-23 are new, and they show that dual matrices have analogous characteristics to those possessed by their real counterparts, when their $\{1\}$-dual generalized inverses exist.

\section{Conclusions}

This paper deals with the question of when a dual matrix $\hat{A}$ has a specified kind of dual generalized inverse. It provides new and foundational results on dual generalized inverses of dual matrices, thereby providing a basic understanding of dual inverses. While all real matrices have a $\{1\}$-dual generalized inverse, all dual matrices may not have a $\{1\}$-dual generalized inverse. The necessary and sufficient condition for many useful dual generalized inverses, such as the $\{1,3\}$ - and $\{1,2,3,4\}$-dual generalized matrices, to exist is found to be simply that a $\{1\}$-dual generalized inverse must exist.

A fairly comprehensive theory of dual generalized inverses is presented. For instance, the formula for the Moore-Penrose dual generalized inverse of a dual matrix whose real part has full row (column) rank has been known for quite some time. What is not known is why this rank condition on the real part arises. Recently, it was also shown, in reference [4], that all dual matrices do not have Moore-Penrose dual generalized inverses (MPDGIs). Such discovery then begs questions, such as, "What kinds of dual matrices 
have Moore-Penrose dual generalized inverses?" and "Can a dual matrix whose real part is rank deficient have a Moore-Penrose dual generalized inverse?" These are questions of practical importance, because such rank deficiency does arise in engineering applications, for example, in the analysis of kinematic systems and mechanisms [4]. The results obtained in this paper answer the latter question in the affirmative. More importantly, they point out that the key to answering such questions requires a redirection of our focus from rank conditions on the real part of a dual matrix to the existence of its $\{1\}$-dual generalized inverse.

The necessary and sufficient condition for a dual matrix to have a $\{1\}$-dual generalized inverse is obtained. It is shown that, for a dual matrix to have a $\{1\}$-dual generalized inverse, its real and dual parts cannot be arbitrarily specified and the dual part of the matrix must depend on its real part. An extension of the result (proof) given in reference [4], which now provides the entire set of $\{1\}$-dual generalized inverses of a dual matrix, is obtained. It is shown that the necessary and sufficient condition for a $\{1\}$-dual inverse to exist is that the dual part of the dual matrix must map the null space of its real part to the column space of its real part.

The paper shows that the necessary and sufficient condition for a dual matrix to have a $\{1,2\}-,\{1,3\},\{1,4\},\{1,2,3\}-,\{1,2,4\}$ and $\{1,2,3,4\}$-dual generalized inverse is that it must have a $\{1\}$-dual inverse. This list covers the most useful dual generalized inverses from an applications viewpoint in science and engineering, such as $\{1,3\}$ - and $\{1,4\}$-dual generalized inverses and the Moore-Penrose dual generalized inverse (MPDGI). Then, what this says is that the existence of a $\{1\}$-dual inverse of a dual matrix is a sine qua non for the existence of all these dual generalized inverses. Moreover, as mentioned earlier, the necessary and sufficient conditions for a dual matrix to have a $\{1\}$-dual generalized inverse are explicitly obtained. In fact, the complete set of $\{1\}$-dual inverses of a dual matrix is explicitly obtained.

Explicit expressions for each of the above-mentioned dual generalized inverses, when they exist, are obtained irrespective of the rank of their real parts, for easy use by scientists and engineers. To the best of the author's knowledge, these explicit expressions have not been reported previously.

It is shown that all dual matrices have $\{2\}$ - and $\{2,4\}$-dual generalized inverses and explicit expressions for these sets of inverses are obtained.

The Moore-Penrose generalized inverse of a dual matrix, in terms of only $\{1\}$-inverses of matrices, is investigated. It is shown that the product matrices $\hat{A} \hat{A}^{T}, \hat{A}^{T} \hat{A}$ and $\hat{A}^{T} \hat{A} \hat{A}^{T}$ always have $\{1\}$-dual generalized inverses. When $\hat{A}$ has a $\{1\}$-dual generalized inverse, explicit expressions for the Moore-Penrose dual generalized inverse of $\hat{A}$, in terms of $\{1\}$-dual inverses of these matrices, are obtained.

It is hoped that the necessary and sufficient conditions developed in this paper, along with the explicit closed form expressions for various types of dual generalized inverses irrespective of the rank of their real parts, will aid in handling practical problems in science and engineering, particularly in the analysis and synthesis of mechanisms and machines, multi-body dynamics, robotic design and trajectory planning and sensor calibration methods. Computational methods in various scientific disciplines for solving both forward and inverse problems will also find the closed form results developed here especially useful, since they provide simple and straightforward approaches for obtaining dual generalized inverses.

Funding: This research received no external funding.

Data Availability Statement: Not applicable.

Conflicts of Interest: The author declares no conflict of interest.

\section{References}

1. Fischer, I. Dual-Number Methods in Kinematics, Statics and Dynamics; CRC Press: Boca Raton, FL, USA, 1998.

2. Condurache, D.; Burlacu, A. Dual Tensors Based Solutions for Rigid Body Motion Parameterization. Mech. Mach. Theory 2014, 74, 390-412. [CrossRef] 
3. Gu, Y.-L.; Luh, J.Y.S. Dual-number Transformations and its Applications to Robotics. IEEE J. Robot. Autom. 1987, 3, 615-623.

4. Udwadia, F.E.; Pennestri, E.; de Falco, D. Do All Dual Matrices have Dual Moore-Penrose Inverses? Mech. Mach. Theory 2020, 151, 103878. [CrossRef]

5. Udwadia, F.E. Dual Generalized Inverses and their Use in Solving Systems of Linear Dual Equations. Mech. Mach. Theory 2021, 156, 104158. [CrossRef]

6. Yanai, H.; Takeuchi, K.; Takane, Y. Projection Matrices, Generalized Inverse Matrices, and Singular Values; Springer: New York, NY, USA, 2011.

7. Udwadia, F.E.; Kalaba, R.E. Analytical Dynamics: A New Approach; Cambridge University Press: Cambridge, UK, 2007.

8. Rao, C.R.; Mitra, S.K. Generalized Inverses and Its Applications; John Wiley: Hoboken, NJ, USA, 1972.

9. Penrose, R. A Generalized Inverse for Matrices. Math. Proc. Camb. Philos. Soc. 1955, 51, 406-413. [CrossRef] 